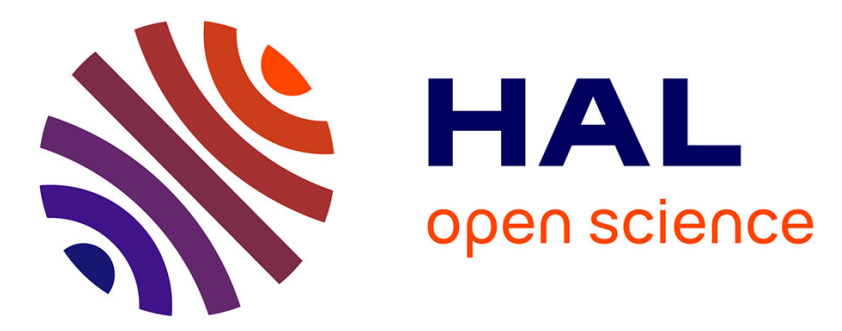

\title{
L'équipement automobile, entre contrainte et distinction sociale
}

Philippe Coulangeon, Ivaylo Petev

\section{To cite this version:}

Philippe Coulangeon, Ivaylo Petev. L'équipement automobile, entre contrainte et distinction sociale. Economie et Statistique / Economics and Statistics, 2012, 457-458, pp.97-121. hal-01053543

\section{HAL Id: hal-01053543 \\ https://hal-sciencespo.archives-ouvertes.fr/hal-01053543}

Submitted on 31 Jul 2014

HAL is a multi-disciplinary open access archive for the deposit and dissemination of scientific research documents, whether they are published or not. The documents may come from teaching and research institutions in France or abroad, or from public or private research centers.
L'archive ouverte pluridisciplinaire HAL, est destinée au dépôt et à la diffusion de documents scientifiques de niveau recherche, publiés ou non, émanant des établissements d'enseignement et de recherche français ou étrangers, des laboratoires publics ou privés. 


\title{
L'équipement automobile, entre contrainte et distinction sociale
}

\author{
Philippe Coulangeon * et Ivaylo D. Petev **
}

Emblématique de la société de consommation et de production de masse, l'automobile connaît en France une diffusion qui, tant du point de vue de l'accès à la motorisation que de la distribution des différentes catégories de véhicules (marque, modèle, puissance, ancienneté et statut d'acquisition), fait apparaître des différences importantes entre les groupes sociaux. Entre ces derniers s'insinuent de nouveaux types de clivages (multimotorisation, progression de la place des marques étrangères, notamment). L'analyse conjointe des habitudes de déplacement et des caractéristiques d'équipement automobile des ménages motorisés en 2008 met en évidence l'articulation d'effets propres aux contraintes de mobilité et aux habitudes de déplacement, qui n'entament pas toutefois la robustesse des écarts entre groupes sociaux. Le statut d'acquisition des véhicules apparaît comme un marqueur social soumis à un cycle de diffusion et de banalisation que révèle l'accès socialement différencié au marché du neuf et de l'occasion. Il se combine au type de véhicule. Les voitures allemandes, en particulier les plus puissantes, apparaissent ainsi comme un marqueur spécifique de l'appartenance aux classes supérieures, en particulier chez les indépendants. Ces écarts soulignent les éléments de compétition statutaire et symbolique qui continuent d'entourer l'acquisition et les usages de l'automobile. 
L' automobile est parfois perçue comme emblématique d'une certaine homogénéisation des modes de vie, corollaire de la massification de la production des biens de consommation et d'équipement des ménages dont s'est accompagnée la croissance des décennies d'après-guerre (Galbraith, 1958 ; Bell, 1973 ; Mendras, 1988). En relâchant l'emprise des contraintes matérielles, cette croissance accentuerait par contraste l'importance des dimensions symboliques de la différenciation sociale des styles de vie (Darras, 1966 ; Bourdieu, 1979), toutefois davantage associées à la consommation des biens immatériels, aux loisirs ou aux pratiques culturelles qu'aux achats de biens d'équipement et aux consommations matérielles (Gartman, 1991). Il est ainsi parfois avancé que l'automobile ne ferait pas spécifiquement l'objet d'un « marketing de classe » (Herpin, 2001), et que la segmentation contemporaine de son marché, marquée par une différenciation fine des gammes de production, principal ressort de la concurrence entre les constructeurs (Panzar et Willig, 1981 ; Teece, 1981 ; Lung et al., 1999), renverrait à une variété et à une volatilité de goûts et de besoins irréductibles aux seuls déterminismes socio-économiques. L'automobile est de ce fait souvent prise comme exemple d'un bien dont les caractéristiques de production en grande série invalideraient les logiques de distinction théorisées par Pierre Bourdieu (Gartman, 2004).

L'automobile n'est cependant pas un bien parfaitement banalisé (Choquet, 1983). Les caractéristiques de l'équipement automobile des ménages sont tout d'abord soumises aux contraintes spatiales et temporelles qui s'exercent sur une population inégalement affectée par les effets de l'étalement urbain et de l'éloignement des lieux de résidence et d'activité, et de ce fait inégalement dépendante à l'usage de l'automobile. Ces caractéristiques sont aussi tributaires de la composition et de la taille des ménages. Celles-ci reflètent les choix et les arbitrages opérés dans la vie familiale ou professionnelle aussi bien qu'en matière de logement. La séquence de ces évènements n'est généralement pas ou peu prise en compte par la plupart des sources disponibles, essentiellement de nature transversale.

En marge de ces contraintes et de ces arbitrages, l'équipement automobile des ménages est aussi soumis à des logiques de distinction sociale ou de compétition statutaire, qui se manifestent notamment dans la segmentation du parc automobile et la distribution des différents types de véhicules (marques, modèles, puissance). Ces dimensions sociales et symboliques ne sont toutefois pas toujours aisément séparables des facteurs évoqués précédemment, tant certaines caractéristiques - volume ou puissance des véhicules, notamment - peuvent aussi bien être tenues pour des éléments d'affirmation statutaire que pour des ressources mobilisées dans la concurrence objective pour l'appropriation de l'espace (Boltanski, 1975 et 1976).

En prenant principalement appui sur les données de l'édition 2008 de l'enquête nationale Transports et déplacements (ENTD) réalisée par l'Insee et l'Institut national de recherche sur les transports et leur sécurité (Inrets) pour le Service de l'Observation et des Statistiques du Ministère de l'Écologie, du Développement Durable, des Transports et du Logement, et, secondairement, sur les éditions précédentes de cette même enquête (1982 et 1994), cette étude se propose d'évaluer ce qui, dans la distribution des caractéristiques de l'équipement automobile des ménages, procède des contraintes fonctionnelles, liées en particulier aux habitudes de déplacement et à la composition des ménages, et ce qui procède davantage de logiques de distinction ou de compétition statutaire entre les groupes sociaux.

\section{La banalisation de l'automobile est allée de pair avec l'apparition de nouveaux clivages sociaux (marques étrangères, état d'acquisition)}

$\mathbf{L}$ 'évolution de l'équipement automobile des ménages français observée au cours des trente dernières années fait apparaître plusieurs évolutions notables. Tout d'abord, la progression des taux de motorisation des ménages est importante (Robin, 2010). Entre 1982 et 2008, la part des ménages non-motorisés (au sens de la possession d'au moins une automobile) est ainsi tombée de 28 à $17 \%$, tandis que celle des ménages multi-motorisés (au moins deux véhicules) passait de 20 à $35 \%$ (cf. graphique I).

Cette évolution globale masque toutefois de grandes disparités selon les catégories sociales. En 2008 comme précédemment en 1994 et 1982, la non-motorisation est rare au sein des catégories supérieures (cadres, chefs 
d'entreprises, professions libérales) - sauf dans l'agglomération parisienne - et demeure beaucoup plus fréquente au sein des classes populaires (cf. graphique II). Entre 1982 et 2008, les écarts entre catégories extrêmes se sont toutefois sensiblement réduits, s'agissant de la non-motorisation comme, en sens inverse, de la multi-motorisation. Les taux d'équipement des catégories supérieures de ménages sont demeurés relativement stables au fil de la période, tandis que la non-motorisation reculait nettement et la multimotorisation progressait fortement au sein des catégories initialement les moins motorisées. La proportion de ménages non-motorisés est ainsi tombée à $18 \%$ en 2008 chez les ouvriers, à $13 \%$ chez les agriculteurs et à $9 \%$ chez les commerçants et artisans. Cette atténuation des écarts entre catégories sociales procède toutefois vraisemblablement pour partie de changements intervenus dans la composition des catégories en question, qu'il s'agisse de la distribution des revenus ou des origines sociales de leurs membres, de la taille des familles, ou, tout simplement, du changement de définition de leur périmètre, comme c'est le cas, en particulier, pour la catégorie des cadres.

Une autre évolution notable du parc automobile des ménages français au cours de la période couverte par la succession des trois enquêtes sur les transports concerne la progression de la part des véhicules de marque étrangère (cf. graphique III) (Méot, 2005 ; Juillard, 2007). Alors qu'en 1982 , seuls $11 \%$ des ménages possédaient au moins un véhicule de marque étrangère, ils sont $21 \%$ dans ce cas en 1994 et $25 \%$ en 2008 . Dans cet ensemble, la progression des véhicules de marque allemande, présents dans $8 \%$ des ménages en fin de période, apparaît particulièrement prononcée. Ces évolutions sont cohérentes avec celles que donnent à voir les indicateurs relatifs aux ventes d'automobiles. La progression des ventes de voitures étrangères, qui représentent aujourd'hui environ $40 \%$ des achats de véhicules neufs en France, soit deux fois plus qu'au début des années 1980 et quatre fois plus qu'au début des années 1960, profite en effet tout particulièrement aux voitures allemandes (Baron, 2002).

Cette internationalisation du marché de l'automobile n'affecte toutefois pas uniformément l'ensemble des ménages français, mais se manifeste plus particulièrement dans les classes supérieures. En 2008, la possession de véhicules de marque allemande s'est ainsi spectaculairement accrue au sein des ménages dont la personne de référence appartient au groupe des

Graphique I

Taux d'équipement et de multi-équipement automobile des ménages en 1981, 1993 et 2008

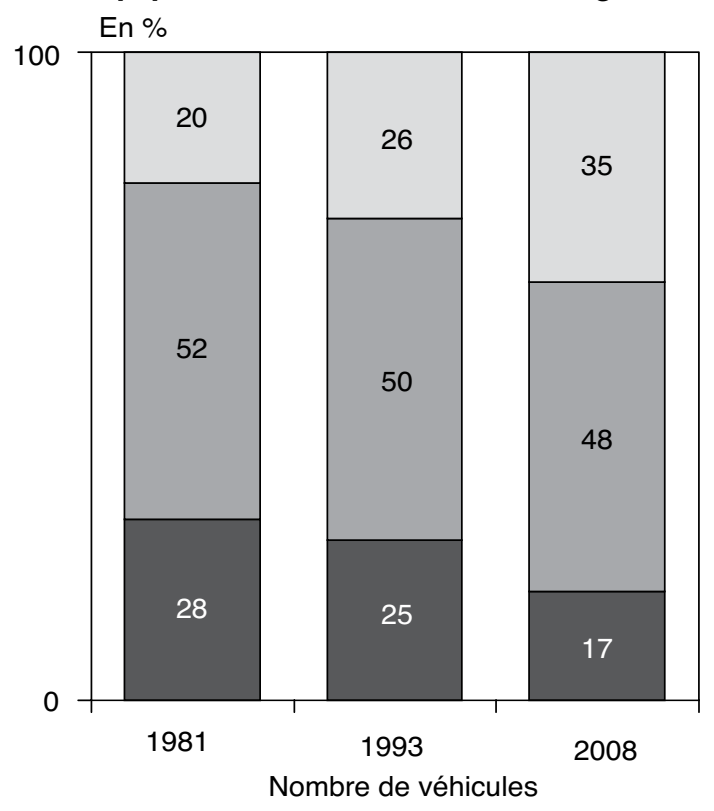

Aucun $\square$ Un $\square$ Deux ou plus

Lecture : en 1981, 28 \% des ménages français ne possédaient aucune automobile.

Champ : ensemble des ménages, France métropolitaine.

Source : enquête Transports (ET) 1981, Enquête Transports et communications (ETC) 1993 et Source : enquête nationale Transports et déplacements (ENTD) 2008, Insee. 
« chefs d'entreprises et professions libérales » par comparaison avec les deux enquêtes antérieures, puisqu'un tiers d'entre eux sont dans ce cas, contre 12 et $17 \%$ en 1981 et 1994 , et elle n'a connu une telle progression dans aucun autre groupe (cf. graphique IV). C'est aussi dans ce groupe que les marques françaises voient leur part se réduire le plus significativement :

\section{Graphique II \\ Taux d'équipement et de multi-équipement automobile selon la catégorie socioprofessionnelle de la personne de référence du ménage de 1981 à 2008}

En \%

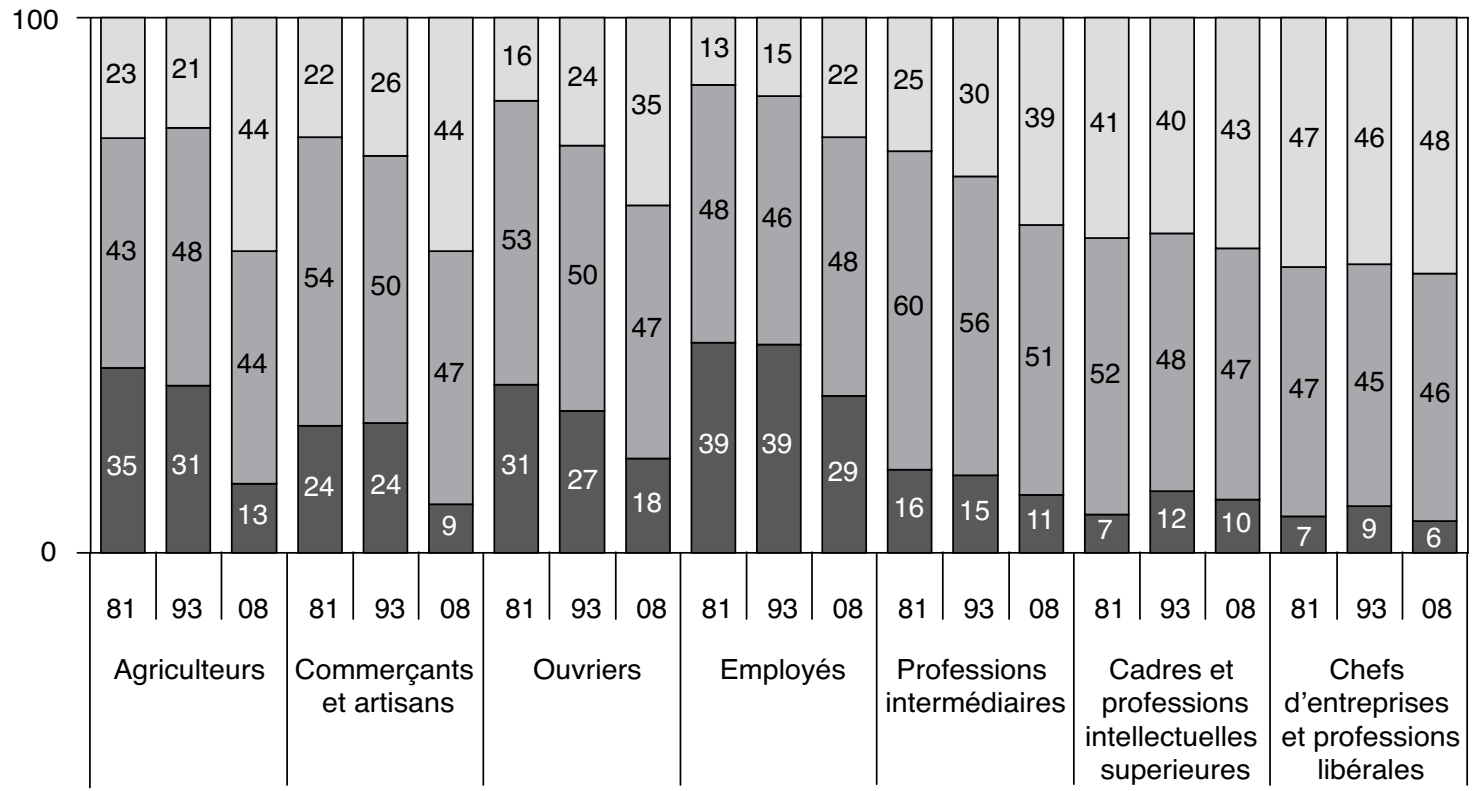

Aucun $\square$ Un $\square$ Deux ou plus

Lecture : en 1981, 31 \% des ménages ouvriers ne possédaient aucune automobile contre $27 \%$ en 1993 et $18 \%$ en 2008.

Champ : ensemble des ménages, France métropolitaine.

Source : enquête Transports (ET) 1981, enquête Transports et communications (ETC) 1993 et enquête nationale Transports et déplacements (ENTD) 2008, Insee.

\section{Graphique III}

Marque et origine des véhicules de 1981 à 2008

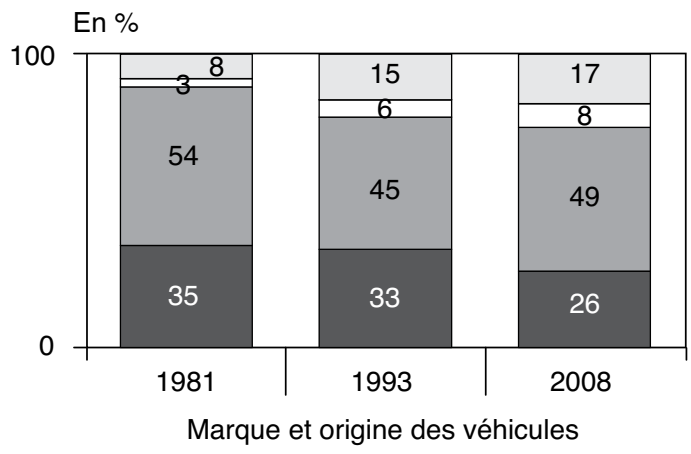

$\square$ Aucun véhicule $\square$ Marques françaises
$\square$ Marques allemandes $\square$ Autres marques
étrangères

Lecture : pour des raisons de comparabilité des champs couverts par les trois enquêtes successives, la distribution des marques et véhicules n'est calculée que sur les ménages possédant au plus un véhicule.

en 1981, $35 \%$ des ménages ne disposaient d'aucun véhicule.

Champ : ménages possédant au plus un véhicule, France métropolitaine.

Source : enquête Transports (ET) 1981, enquête Transports et communications (ETC) 1993 et enquête nationale Transports et déplacements (ENTD) 2008, Insee. 
alors qu'en 1982, $60 \%$ des ménages de cette catégorie possédaient un véhicule d'un des trois grands constructeurs français, ils ne sont plus que $38 \%$ dans ce cas en $2008^{1}$.

À côté de la distribution des véhicules de marque française et étrangère - en particulier allemande - , l'état d'acquisition des véhicules possédés au sein des ménages apparaît aussi particulièrement clivant. Ainsi, la possession de véhicules achetés neufs n'est-elle en 2008 majoritaire que parmi les ménages de chefs d'entreprises et de professions libérales, tandis qu'un peu moins de la moitié des ménages de cadres et professions intellectuelles sont dans ce cas, et moins d'un quart des ménages ouvriers (cf. tableau 1).

Pour autant, la part du neuf et de l'occasion varie selon le type de véhicule possédé. Ainsi, pour l'année 2008, le croisement des types de véhicules et de leur état d'acquisition, fondé sur une nomenclature des véhicules combinant marque et puissance fiscale, qui permet de tenir compte de l'étendue de la gamme des modèles en distinguant les véhicules d'une puissance au moins égale à $6 \mathrm{cv}$ fiscaux - valeur médiane de la puissance observée au sein du parc automobile des ménages - des véhicules de moindre puissance, fait apparaître que certains types de véhicules, majoritairement acquis à l'état neuf dans les catégories supérieures (cadres supérieurs et chefs d'entreprises), le sont majoritairement à l'état d'occasion dans les ménages ouvriers. Il en va ainsi plus particulièrement des véhicules de marque française de puissance supérieure à $6 \mathrm{cv}$, ainsi que des voitures allemandes de même puissance (cf. tableau 2).

Si l'accès socialement différencié au marché des véhicules neufs et d'occasion traduit sans aucun doute l'effet sous-jacent de la contrainte budgétaire qui s'exerce sur les différentes catégories de ménages, le fait que cette différenciation

1. Pour neutraliser les effets de la progression de la multi-motorisation au fil des enquêtes, la distribution des marques de voitures ne porte ici que sur les ménages ne possédant qu'un seul véhicule automobile, qui représentent à peu près les deux tiers des ménages motorisés. La majorité (environ 80 \%) du tiers restant se compose de ménages dotés d'au moins deux véhicules, dont la distribution par marques ne diffère pas fondamentalement de celle observée aux graphiques I et IV.

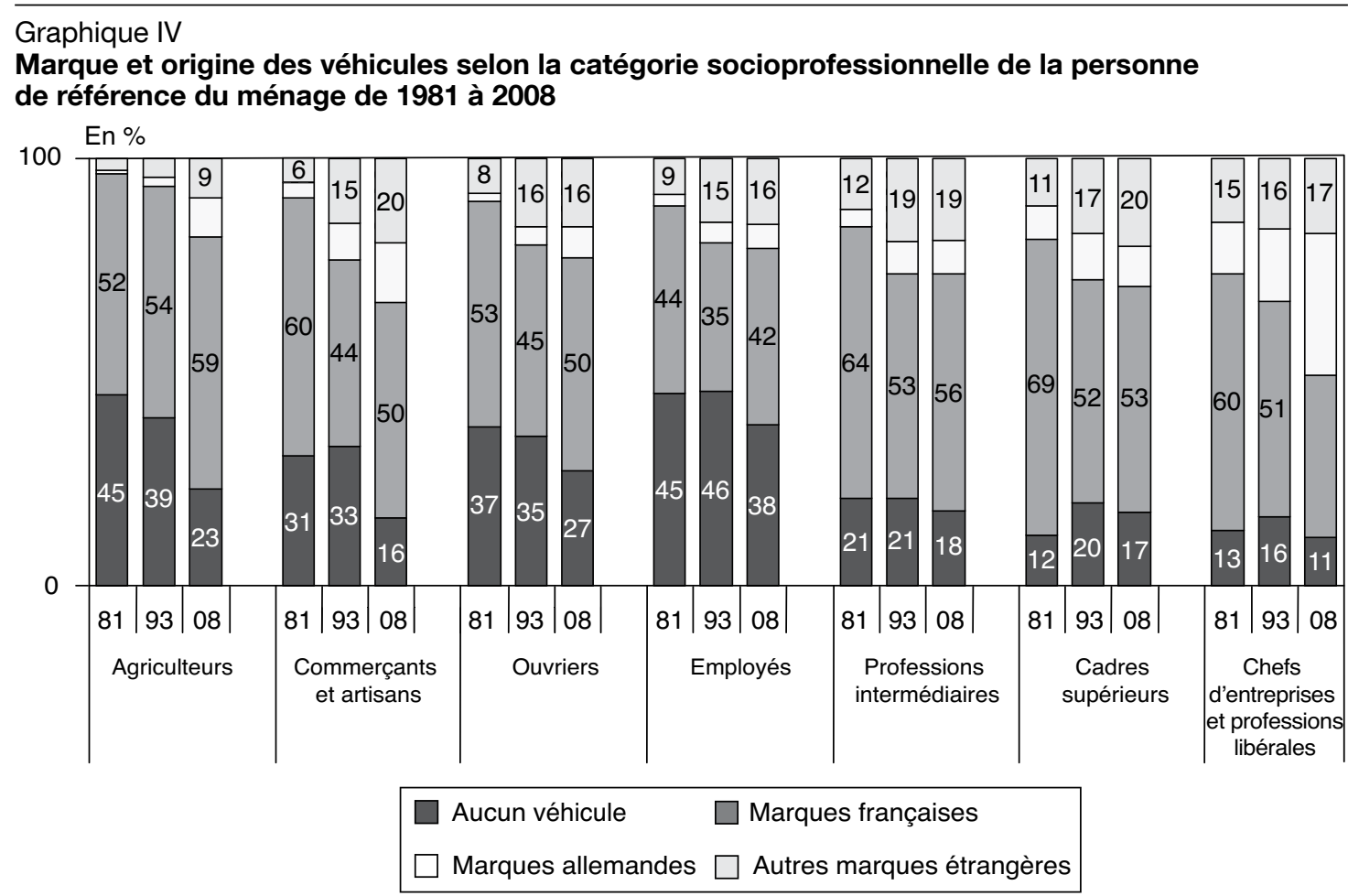

Lecture : pour des raisons de comparabilité des champs couverts par les trois enquêtes successives, la distribution des marques et véhicules n'est calculée que sur les ménages possédant au plus un véhicule.

En 1981, 45 \% des ménages d'agriculteurs ne possédaient aucun véhicule.

Champ : ménages possédant au plus un véhicule, France métropolitaine.

Source : enquête Transports (ET) 1981, enquête Transports et communications (ETC) 1993 et enquête nationale Transports et déplacements (ENTD) 2008, Insee. 
n'opère pas au même degré selon les marques et la puissance des véhicules incite aussi à lire l'articulation du marché du neuf et de l'occasion comme la manifestation de logiques de diffusion et de dévaluation du pouvoir de distinction sociale des véhicules les plus puissants et les plus onéreux lorsque ceux-ci sont acquis en seconde main. Ces logiques de diffusion évoquent pour partie celles rencontrées dans la diffusion d'autres catégories de biens matériels ou symboliques (Bourdieu, 1979), comme le montre le cas bien connu des prénoms (Besnard et Desplanques, 1999 ; Lieberson, 2000 ; Coulmont, 2011), cas limite de biens totalement libres, pour lesquels les choix opérés ne sont soumis à aucune contrainte de revenu ou de prix. La part des effets de valorisation/dévalorisation symbolique est inévitablement moindre et les choix plus nettement subordonnés à l'effet des contraintes de budget dans le cas de biens tels que les biens automobiles.

Les transformations du parc automobile des ménages français peuvent aussi être rapprochées des conséquences de l'évolution des structures résidentielles, en particulier de la progression et de l'allongement des déplacements quotidiens liés à l'étalement des grandes métropoles (Le Jeannic, 1997 ; Hubert et Delisle, 2010). Centrée sur les données de l'ENTD 2008, la suite de cet article explore plus précisément les facteurs sociaux de l'équipement automobile et de ses caractéristiques, en s'interrogeant en premier lieu sur le degré auquel celles-ci sont liées aux contraintes associées aux habitudes de déplacement.

Tableau 1

Proportion de ménages disposant d'au moins un véhicule neuf selon le groupe socioprofessionnel de la personne de référence

\begin{tabular}{|l|c|}
\hline \multicolumn{1}{|c|}{ Groupe socioprofessionnel de la personne de référence } & Proportion de ménages disposant d'au moins un véhicule neuf \\
\hline Agriculteurs & 34,8 \\
Artisans et commerçants & 39,3 \\
Chefs d'entreprises et professions libérales & 55,9 \\
Cadres et professions intellectuelles supérieures & 47,8 \\
Professions intermédiaires & 38,0 \\
Employés & 29,1 \\
Ouvriers & 24,0 \\
& \\
Ensemble & 33,3 \\
\hline
\end{tabular}

Lecture : 56 \% des ménages dont la personne de référence appartient au groupe socioprofessionnel des chefs d'entreprises et professions libérales disposent d'au moins un véhicule neuf contrairement à un tiers de l'ensemble des ménages en 2008.

Champ : ménages disposant d'au moins un véhicule neuf, France métropolitaine.

Source : enquête nationale Transports et déplacements (ENTD) 2008, Insee.

Tableau 2

Part des véhicules acquis à l'état neuf par catégorie dans les ménages ouvriers et les ménages de cadres supérieurs, chefs d'entreprises et professions libérales en 2008

\begin{tabular}{|l|c|c|c|}
\hline & Cadres supérieurs, professions libérales, chefs d'entreprises & Ouvriers & Ensemble \\
\hline Marques françaises $\leq 6 \mathrm{cv}$ & 45,7 & 28,5 & 37,1 \\
Marques françaises > $6 \mathrm{cv}$ & 52,7 & 20,0 & 34,1 \\
Marques allemandes $\leq 6 \mathrm{cv}$ & 47,9 & 28,0 & 34,1 \\
Marques allemandes > $6 \mathrm{cv}$ & 47,5 & 16,2 & 36,4 \\
Autres marques étrangères $\leq 6 \mathrm{cv}$ & 53,6 & 34,5 & 44,5 \\
Autres marques étrangères > $6 \mathrm{cv}$ & 55,3 & 36,0 & 45,3 \\
\hline
\end{tabular}

Lecture : 45,7 \% des ménages de cadres supérieurs, professions libérales ou chefs d'entreprises possédant au moins un véhicule de marque française de puissance fiscale inférieure à $6 \mathrm{cv}$ en possèdent au moins un acquis à l'état neuf. 28,5\% des ménages ouvriers sont dans ce cas.

Champ : ménages d'ouvriers et de cadres supérieurs, chefs d'entreprises et professions libérales possédant au moins un véhicule automobile.

Source : enquête nationale Transports et déplacements (ENTD) 2008, Insee. 


\section{Les facteurs sociaux des habitudes de déplacement et de l'équipement automobile}

L e rapprochement des caractéristiques de l'équipement automobile de celles des habitudes de déplacement articule deux niveaux d'analyse distincts : celui des ménages et celui des individus. Les évolutions relevées au niveau des ménages occultent ainsi en partie la progression de formes plus nettement individualisées d'appropriation et d'usage de l'automobile, qui vont de pair avec la multimotorisation observée en fin de période. Dans la mesure où les données de l'ENTD permettent d'attribuer les véhicules recensés à leurs conducteurs habituels, les observations précédentes peuvent être précisées en associant les véhicules possédés aux propriétés de leurs conducteurs. Aux caractéristiques sociodémographiques d'âge, de sexe, de statut d'activité, de catégorie socioprofessionnelle, de revenu, de taille et de composition du ménage, s'ajoutent ici les données relatives aux habitudes de déplacement, elles-mêmes dépendantes des propriétés de l'environnement de résidence (densité, éloignement des lieux de vie et d'activité, etc.). La caractérisation des habitudes de déplacement met aussi en jeu la balance des déplacements locaux et des déplacements de longue distance, que l'enquête renseigne séparément.

\section{L'espace social des transports et déplacements au sein des ménages motorisés}

Afin de relier la caractérisation des habitudes de déplacement de celle des véhicules utilisés, l'analyse est restreinte à une population susceptible de faire usage de véhicules automobiles, à savoir l'ensemble de la population adulte titulaire du permis B dont les déplacements ont été renseignés de manière détaillée au cours de la deuxième visite de l'enquête et appartenant à des ménages possédant au moins un véhicule automobile (cf. encadré) ${ }^{2}$. Le rapprochement des caractéristiques de l'équipement automobile et des habitudes de déplacement laisse ainsi volontairement de côté la population des ménages non-motorisés.

On procède sur les 13216 individus retenus à une analyse des correspondances multiples (ACM) dans laquelle les individus sont caractérisés par huit variables actives qui décrivent leurs habitudes en matière de déplacement. Deux d'entre elles sont plus spécifiquement relatives aux usages de l'automobile. La première, qui concerne la mobilité quotidienne, totalise la distance cumulée des déplacements automobiles locaux et distingue les individus selon qu'ils ont parcouru plus ou moins de $10 \mathrm{~km}$ à l'intérieur d'un cercle de $80 \mathrm{~km}$ autour du domicile au cours de la semaine de référence de l'enquête. La deuxième variable partage la population enquêtée selon la présence ou l'absence de déplacements automobiles à

\footnotetext{
2. La restriction aux seuls titulaires $d u$ permis $B$ pose un problème de sélection endogène que nous avons choisi ici de laisser de côté, en privilégiant simplement l'homogénéité de la population prise en considération. De même, la restriction aux personnes appartenant à des ménages possédant au moins un véhicule automobile laisse de côté celles qui se passent volontairement de véhicule personnel, préférant recourir aux services de taxis ou aux agences de location, qui correspondent, dans l'échantillon, à un nombre d'individus très réduit.
}

Encadré

\section{POPULATION COUVERTE PAR L'ANALYSE : INDIVIDUS ET VÉHICULES}

La population couverte par l'analyse est déterminée par l'agencement des tables de l'ENTD issues des deux visites successives sur lesquelles s'appuie l'enquête. Pour disposer d'informations détaillées sur les habitudes de transports, on restreint l'analyse aux individus Kish, soit un individu par ménage, tiré au sort pour répondre au questionnaire de la deuxième visite, où sont renseignées les tables de mobilité, de déplacements locaux et de voyages à longue distance. Une seconde restriction est imposée en ne conservant que les individus susceptibles de faire usage d'une automobile, que l'on définit ici comme l'ensemble des personnes adultes titulaires du permis B et appartenant à des ménages dotés d'au moins un véhicule automobile.

Du point de vue des caractéristiques de l'équipement automobile, le nombre total de véhicules présents dans le ménage est pris en compte, mais, les usages sont renseignés seulement sur la base des véhicules qui peuvent être appariés à leurs conducteurs, soit deux au plus pour chaque ménage, du fait des contraintes imposées pour la $2^{\text {ème }}$ visite de l'enquête. L'analyse articule donc les niveaux ménage et individu en attribuant les véhicules à leurs conducteurs, étant entendu qu'à l'intérieur d'un ménage, un même véhicule peut être conduit par plusieurs personnes et que, pour les ménages - très minoritaires - disposant de plus de deux véhicules, l'information est lacunaire pour les véhicules au-delà du deuxième. Mais l'unité d'analyse demeure bien l'individu Kish tiré au sort dans chaque ménage, et c'est la variable de pondération correspondante qui est utilisée (PONDKI). 
longue distance au cours des 13 semaines précédant l'enquête ( $2^{\text {ème }}$ visite, cf. encadré). Cinq variables indicatrices permettent par ailleurs de particulariser les individus qui, au cours de l'année précédant l'enquête, ont effectué au moins un voyage à plus de $100 \mathrm{~km}$ du domicile en train, ont pris au moins une fois l'avion, sont partis en vacances, ont utilisé au moins une fois un moyen de transport en commun, et ont effectué des trajets de proximité par d'autres moyens que l'automobile au cours de la semaine de référence renseignée au cours de la seconde visite. Enfin, une dernière variable indique si la personne enquêtée effectue au moins 30 minutes de marche à pied par jour.

Le choix de ces variables permet de caractériser les habitudes de transport en tenant compte simultanément du volume, de la temporalité, de l'échelle et des modalités de déplacement. Les deux premières dimensions extraites par l'ACM, qui captent plus de $40,30 \%$ de la variance totale du nuage de points, synthétisent l'essentiel des principes de dispersion des attitudes observées en la matière (cf. graphique V).

Le premier axe, qui totalise un peu plus de $24 \%$ de la variance, est principalement construit par les variables d'accès à la mobilité longue distance (départ en vacances, voyages en avion et en train à plus de 100 kilomètres du domicile) et de recours aux modes de transports quotidiens alternatifs à l'automobile. Il oppose, de gauche à droite, les personnes qui sont parties en vacances au cours de l'année précédant l'enquête à celles qui ne sont pas parties, celles qui ont emprunté au moins une fois le train ou l'avion à celles qui n'ont pas emprunté ces moyens de transport, les usagers aux non-usagers des transports en commun et des modes de déplacement quotidiens alternatifs à l'automobile, dont la pratique semble ainsi corrélée à l'accès à la mobilité longue distance. Voyager loin au cours de l'année semble aller de pair avec le fait de recourir moins exclusivement à l'automobile au quotidien et de se déplacer davantage par des transports en commun.

Ce lien entre les caractéristiques des déplacements de proximité et des déplacements à longue distance peut être rapproché des propriétés associées aux variables supplémentaires introduites dans l'analyse qui semblent le plus fortement liées à cet $\mathrm{axe}^{3}$, notamment la taille de l'unité urbaine de résidence, qui isole plus particulièrement les habitants de l'unité urbaine de Paris, caractérisés par un accès privilégié à la mobilité longue distance et aux modes de transport alternatifs à l'automobile ${ }^{4}$. Plus largement, il semble, au vu de la position des points moyens des différentes tailles d'unités urbaines, que l'accès à la mobilité longue distance et aux modes de transports alternatifs à l'automobile s'accroisse avec la taille et la densité du lieu de résidence, cet effet ne se réduisant pas au contraste Paris/province ${ }^{5}$.

Ce premier axe apparaît aussi fortement structuré par les variables de revenu et de catégorie socioprofessionnelle. La projection des déciles de revenu par unité de consommation du ménage suggère ainsi une corrélation positive de l'accès à la mobilité longue distance avec le niveau des ressources économiques. De même, l'accès à la mobilité à longue distance semble t-il affecté d'un fort gradient social qui oppose, du côté des plus mobiles, les cadres et professions intellectuelles supérieures ainsi que les chefs d'entreprises et les professions libérales, aux ouvriers et aux agriculteurs, du côté des moins mobiles - que ces personnes soient encore en activité ou non, comme le suggère la proximité des points moyens des actifs et des ex-actifs de chaque catégorie que distingue le codage retenu pour la catégorie socioprofessionnelle ${ }^{6}$.

Le deuxième axe, qui absorbe un peu plus de $15 \%$ de la variance, apparait essentiellement défini par l'intensité des usages de l'automobile, puisqu'il oppose, de bas en haut, les faibles kilométrages en voiture (en longue distance comme en déplacement de proximité), l'usage, dans la mobilité quotidienne de proximité, des transports en commun et des modes alternatifs à l'automobile, ainsi que l'absence de départ en vacances, d'un côté, aux kilométrages automobiles élevés, de l'autre. Du point de vue des caractéristiques introduites en variables supplémentaires, le deuxième axe est fortement structuré par l'âge, plus précisément par l'opposition entre les plus de 60 ans et les autres, ce que confirme la position de part et d'autres

3. On s'appuie ici sur le critère du ratio de la variance des scores factoriels entre catégories de la variable supplémentaire considérée sur la somme des variances intra et intercatégories de cette même variable ( $\eta^{2}$ ) (Le Roux et Rouanet, 2004).

4. L'unité urbaine de Paris comporte un ensemble de $396 \mathrm{com}$ munes qui couvre la totalité des départements des Hauts-deSeine, Seine-Saint-Denis et Val-de-Marne et une partie des départements de l'Essonne, du Val d'Oise, de la Seine et Marne et des Yvelines. La construction de ce premier axe n'apparaît donc pas comme un artefact lié aux parisiens « intra-muros » qui ne sont pas isolés en tant que tel.

5. La réplication de l'ACM en excluant les résidants de l'unité urbaine de Paris fait apparaitre une structuration homologue des axes.

6. Le classement des individus s'opère en fonction de la dernière activité connue. Contrairement à l'usage, les professions libérales sont ici regroupées avec les chefs d'entreprises et non avec les cadres et professions intellectuelles supérieures. 
de l'axe des actifs occupés, d'une part, des inactifs et ex-actifs, d'autre part : les ouvriers (cps6) s'opposent ainsi davantage sur cet axe aux anciens ouvriers (EXcps6) qu'aux cadres (cps3), qui eux-mêmes s'opposent davantage aux ex-cadres (EXcps3) qu'aux autres catégories d'actifs. Les ressorts de la différenciation sociale des habitudes quotidiennes de déplacement de proximité et des comportements de mobilité en longue distance ne semblent donc pas exactement de même nature. Alors que la différenciation s'opère plutôt dans un cas au regard de l'avancée en âge, du statut d'activité et des contraintes qui y sont associées, elle relève davantage dans l'autre d'une différenciation entre groupes sociaux proprement dits, sans qu'il soit possible de distinguer par cette seule analyse factorielle ce qui relève de l'effet des différences de revenu de ce qui relève de différences socioculturelles inscrites dans des styles de vie spécifiques. L'exploration des facteurs suivants, qui absorbent une part rapidement

\section{Graphique V}

\section{Habitudes de déplacement : ACM, plan des deux premiers facteurs}

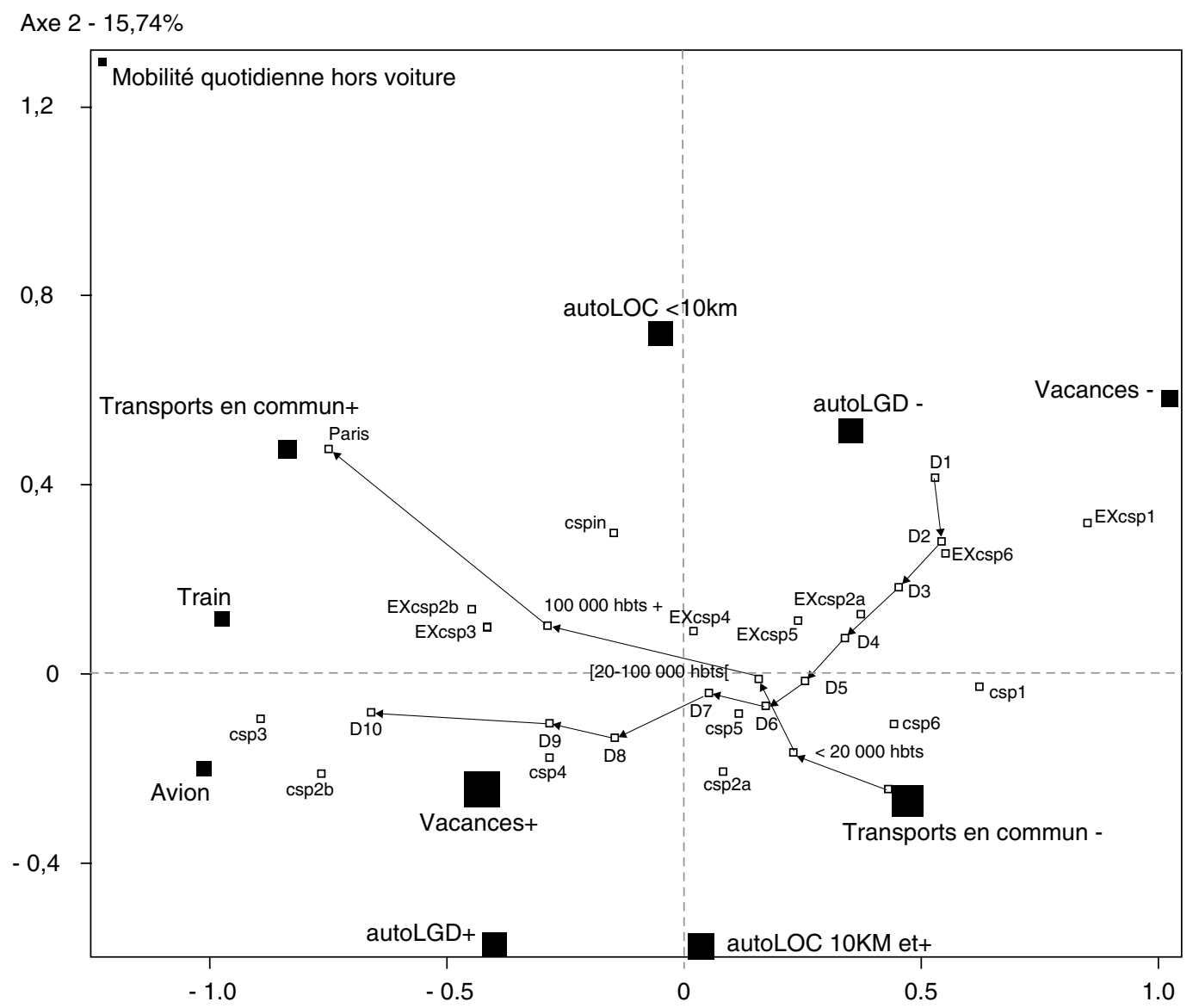

Axe $1-24,56 \%$

Lecture : selon un usage établi en analyse des correspondances multiples, seules sont représentées dans le plan formé par les deux premiers facteurs les modalités présentant des contributions supérieures à la moyenne des contributions sur au moins l'un des deux axes. Les modalités correspondant à une dimension explicative continue sont toutes représentées et reliées. La variable autoLOC désigne les déplacements automobiles en mobilité quotidienne de proximité (moins de $80 \mathrm{~km}$ ). La variable autoLGD désigne les déplacements automobiles sur longue distance (plus de $80 \mathrm{~km}$ ). Les signes + et - désignent les modalités 1 et 0 des variables dichotomiques correspondantes. Pour les déplacements en train et avion, seules les modalités 1 sont représentées, d'où l'absence de signe +. Le suffixe EX désigne la catégorie socioprofessionnelle de la dernière profession exercée par les ex-actifs. Les catégories socioprofessionnelles sont représentées par les abréviations suivantes :

csp1: Agriculteurs

csp2a : Artisans et commerçants

csp2b : Chefs d'entreprises et professions libérales

csp3 : Cadres supérieurs

csp4: Professions intermédiaires

csp5: Employés

csp6: Ouvriers

cspln : Étudiants et sans activités (exclu)

Les carrés représentant les modalités des variables actives ont une taille proportionnelle à la taille relative des effectifs de ces modalités. Champ : ménages possédant au moins un véhicule automobile.

Source : enquête nationale Transports et déplacements (ENTD) 2008, Insee. 
décroissante de la variance du nuage des points, fait apparaître des principes de différenciation additionnels, notamment l'opposition entre mobilité locale et mobilité longue distance, qui permettent d'affiner la caractérisation des habitudes de déplacements.

\section{Au regard des déplacements, les personnes motorisées se répartissent en trois catégories nettement typées}

La structuration de l'espace des habitudes de transports et déplacements peut être précisée en appliquant un algorithme de classification ascendante hiérarchique aux scores des individus sur l'ensemble des facteurs extraits par l'ACM (méthode de Ward). La partition en trois classes, dont les ellipses de concentration sont projetées dans le plan des deux premiers facteurs, et qui satisfait au critère de maximisation de la variance interclasses et de minimisation de la variance intraclasses, fait ressortir trois profils distincts de comportements en matière de transports et de déplacements (cf. graphique VI).

\section{Profil 1 : les hypo-mobiles}

Le premier profil, qui représente $28,2 \%$ des répondants, correspond à des personnes très

\section{Graphique VI}

Habitudes de déplacement : ACM, projection des ellipses de concentration des trois profils de mobilité et déplacements dans le plan des deux premiers facteurs

Axe 2

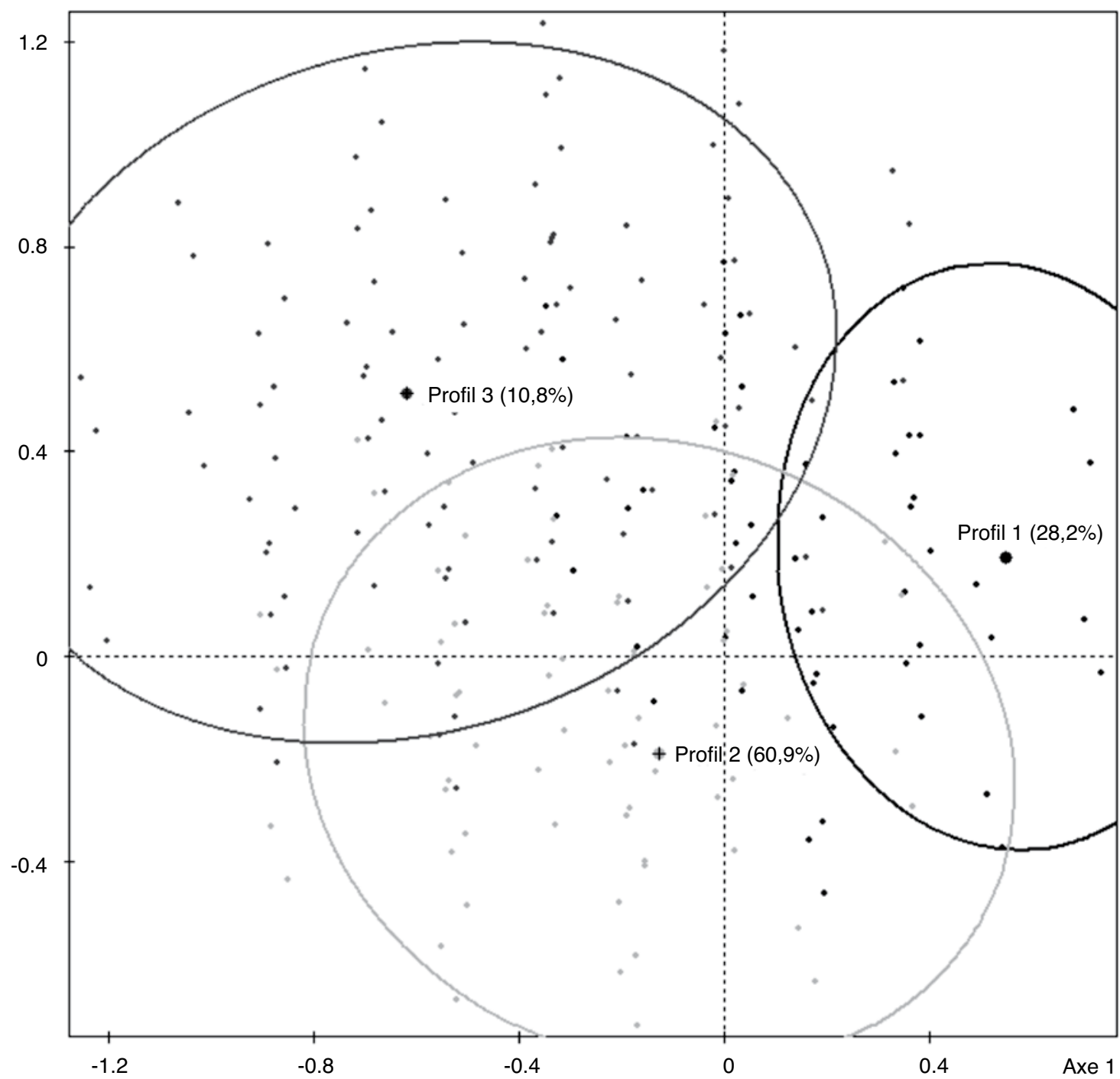

Lecture : $28 \%$ des ménages appartiennent au profil 1 (hypo-mobiles), $61 \%$ au profil 2 (hyper-mobiles à dominante automobile) et $11 \%$ au profil 3 (hyper-mobiles à dominante transports en commun).

Champ : ménages possédant au moins un véhicule automobile, France métropolitaine

Source : enquête nationale Transports et déplacements (ENTD) 2008, Insee. 
peu mobiles, qu'il s'agisse de mobilité locale quotidienne (trajets de moins de $80 \mathrm{~km}$ ) ou de mobilité à longue distance (cf. tableau 3). Une majorité des répondants correspondants à ce profil déclarent ainsi en mobilité locale des kilométrages automobiles inférieurs à 10 $\mathrm{km}$ au cours de la semaine de référence de l'enquête et, pour la quasi-totalité d'entre eux, ne font état d'aucun déplacement en mobilité locale par des modes de transports alternatifs à l'automobile. Ils sont même, au regard de 1'ensemble des répondants, moins nombreux, quoique majoritaires (53\%), à déclarer 30 minutes de marche quotidienne, et utilisent sensiblement moins les transports en commun que la moyenne : ils sont 18,2\% seulement à déclarer y avoir eu recours au moins une fois au cours de l'année précédant l'enquête, soit nettement moins que les répondants des deux autres profils. Cette mobilité réduite à l'échelle des déplacements quotidiens de proximité n'est aucunement compensée par une mobilité plus forte en longue distance, puisque c'est dans ce profil, où l'on enregistre aucun départ en vacances au cours de l'année précédant l'enquête, que l'occurrence des déplacements en train $(11,8 \%)$ et plus encore en avion $(2,6 \%)$ est aussi la plus faible.

\section{Profil 2 : des hyper-mobiles à dominante automobile}

Le deuxième profil, qui concentre la plus grande partie de la population $(60,9 \%)$, apparaît doublement caractérisé par une forte mobilité et un recours plus prononcé aux déplacements automobiles. Une majorité des répondants de ce deuxième profil renseignent ainsi des déplacements de cette nature en mobilité locale quotidienne comme en longue distance (respectivement 58,6 et 57,6 \%). Par ailleurs, la propension aux déplacements en avion (31,6\%), en train $(31,8 \%)$ et, plus largement, à l'usage des transports en commun $(35,2 \%)$, quoique minoritaire, y est plus prononcée que dans la population prise dans son ensemble. Enfin, la quasi-totalité des personnes répondant à ce profil fait état d'au moins un départ en vacances dans l'année précédant l'enquête, contrastant nettement de ce point de vue avec le profil précédent.

\section{Profil 3 : les hyper-mobiles \\ à dominante transports en commun}

Le troisième et dernier profil, qui concerne une proportion nettement plus réduite que les

Tableau 3

Caractéristiques des habitudes de déplacement selon les profils de mobilité (en \%)

\begin{tabular}{|c|c|c|c|c|}
\hline & $\begin{array}{c}\text { Profil } 1 \\
\text { Hypo-mobiles }\end{array}$ & $\begin{array}{c}\text { Profil } 2 \\
\text { Hyper-mobiles } \\
\text { à dominante } \\
\text { automobile }\end{array}$ & $\begin{array}{c}\text { Profil } 3 \\
\text { Hyper-mobiles } \\
\text { à dominante transports } \\
\text { en commun }\end{array}$ & Ensemble \\
\hline $\begin{array}{l}\text { Ont pris l'avion au moins une fois au cours } \\
\text { de l'année }\end{array}$ & 2,6 & 31,6 & 36,3 & 23,9 \\
\hline $\begin{array}{l}\text { Ont pris le train au moins une fois au cours } \\
\text { de l'année précédant l'enquête }\end{array}$ & 11,8 & 31,8 & 43,8 & 27,5 \\
\hline $\begin{array}{l}\text { Sont partis en vacances au cours de l'année } \\
\text { précédant l'enquête }\end{array}$ & 0,0 & 99,6 & 82,4 & 69,7 \\
\hline Marchent au moins 30 minutes par jour & 53,4 & 56,6 & 66,2 & 56,7 \\
\hline $\begin{array}{l}\text { Utilisation des transports en commun au cours } \\
\text { de l'année }\end{array}$ & 18,2 & 35,2 & 67,5 & 33,9 \\
\hline $\begin{array}{l}\text { Déplacements quotidiens par d'autres moyens } \\
\text { que l'automobile }\end{array}$ & 0,5 & 0,0 & 100,0 & 11,0 \\
\hline \multicolumn{5}{|l|}{$\begin{array}{l}\text { Déplacements automobiles en mobilité locale } \\
\text { au cours de la semaine de référence: }\end{array}$} \\
\hline moins de $10 \mathrm{~km}$ & 51,2 & 41,5 & 62,2 & 46,5 \\
\hline $10 \mathrm{~km}$ ou plus & 48,8 & 58,6 & 37,8 & 53,6 \\
\hline $\begin{array}{l}\text { Déplacements automobiles en longue distance } \\
\text { au cours des } 13 \text { semaines précédant l'enquête }\end{array}$ & 22,8 & 57,6 & 51,7 & 47,1 \\
\hline
\end{tabular}

Lecture : parmi les ménages hypo-mobiles, 0,5\% déclare des déplacements quotidiens par d'autres moyens que l'automobile, 48,8 \% déclarent des déplacements automobiles en mobilité locale de $10 \mathrm{~km}$ ou plus, et 22,8\% déclarent des déplacements automobiles en longue distance.

Champ : ménages possédant au moins un véhicule automobil, France métropolitaine.

Source : enquête nationale Transports et déplacements (ENTD) 2008, Insee. 
deux précédents $(10,8 \%)$, fait ressortir le cas particulier d'individus très mobiles, localement comme sur longue distance, mais dont la mobilité, en particulier localement, s'avère nettement moins dépendante de l'automobile que pour le profil précédent. 62,2\% des répondants entrant dans ce profil renseignent ainsi des déplacements automobiles locaux inférieurs à 10 kilomètres au cours de la semaine de référence de l'enquête, et ils renseignent tous par ailleurs des trajets effectués par d'autres modes de transports que l'automobile. C'est aussi dans ce profil que le recours aux transports en commun est le plus fréquent $(67,5 \% \mathrm{y}$ ont eu recours au cours de l'année précédant l'enquête). Sur la longue distance, toutefois, la voiture s'avère d'un usage relativement fréquent $(51,7 \%$ des répondants correspondant à ce profil déclarent des déplacements automobiles en longue distance au cours des treize semaines précédant l'enquête). Par ailleurs, si les départs en vacances y sont un petit moins nombreux que dans le profil précédent, les déplacements en avion et en train y sont sensiblement plus fréquents que dans le reste de la population : la mobilité sur longue distance apparait globalement plus importante dans cette catégorie que dans les deux autres.

La position des ellipses de concentration des trois profils dans le plan formé par les deux premiers facteurs de l'ACM (cf. graphique VI) suggère aussi que la distribution des habitudes de déplacement puisse être liée à certaines caractéristiques sociodémographiques des répondants (cf. annexe). On s'attend ainsi à ce que les habitants des communes rurales et des villes petites et moyennes se rencontrent davantage dans le premier profil, les Parisiens et les habitants des grandes métropoles, dans le troisième, les actifs davantage dans le deuxième que dans les deux autres. Du fait de la forte structuration du premier axe par la variable de catégorie socioprofessionnelle, on s'attend aussi à ce que les agriculteurs, les inactifs n'ayant jamais travaillé et les ouvriers soient plus nombreux dans le premier profil que dans les deux autres, et à ce que les catégories moyennes et supérieures du salariat, les professions libérales et les chefs d'entreprises soient sensiblement surreprésentées dans le deuxième et le troisième. La séparation des effets imputables à ces différentes caractéristiques au moyen de régressions logistiques des probabilités d'appartenance à chacun des trois profils permet de préciser les facteurs sociaux de cette distribution (cf. tableau 4).

\section{Les rapports à la mobilité ne dépendent pas seulement de sa compatibilité avec l'activité professionnelle, mais aussi d'une « motilité » intrinsèque propre à chaque catégorie socioprofessionnelle}

La probabilité d'appartenir au premier profil apparaît fortement, et négativement, associée au revenu ${ }^{7}$, au niveau d'éducation, à la taille du ménage et de l'unité urbaine de résidence, et positivement corrélée à l'âge, en sorte que la faible mobilité associée à ce profil apparaît bien comme un attribut de l'isolement, de la pauvreté monétaire, de la ruralité et de l'avancée en âge. Elle sépare aussi très nettement les ouvriers des cadres supérieurs. De même, la probabilité d'appartenir au deuxième profil, qui apparaît fortement liée au revenu, à la taille du ménage et au statut d'activité (cette probabilité est fortement réduite pour les personnes retirées de l'emploi), qui sépare aussi très nettement les Parisiens des habitants d'autres agglomérations de plus de 100000 habitants (avec un effet positif pour les seconds et négatif pour les premiers), est, elle aussi, affectée par la catégorie professionnelle, puisqu'un effet positif significatif est associé à la catégorie des professions intermédiaires.

L'effet de la variable catégorie socioprofessionnelle s'avère plus limité pour le troisième profil, puisqu'il ne distingue significativement que les agriculteurs. Cela suggère que les habitudes de déplacement ne sont pas totalement contraintes par les facteurs de revenu, de localisation, d'âge, de statut d'activité ou de taille du ménage, mais qu'elles distinguent aussi des styles de vie spécifiques à certains groupes sociaux. Ces différences renvoient sans doute pour partie à des caractéristiques de l'activité professionnelle non captées par les autres variables. Elles témoignent aussi, plus globalement, de rapports à la mobilité socialement et culturellement différenciés. Ainsi de l'effet fortement séparateur des modalités « cadres » et « ouvriers » pour le premier profil : il peut s'expliquer par des activités professionnelles très inégalement compatibles

\footnotetext{
7. Dans chacun des modèles, l'effet du revenu est introduit sous la forme du Log du revenu. Une spécification alternative, incluant un terme quadratique, non reproduite ici, ne modifie ni le signe ni l'ampleur du paramètre associé au log du revenu ni celui des autres paramètres, et l'effet associé au terme quadratique luimême n'apparaît pas significatif. La même démarche a été adoptée pour les modèles présentés dans les tableaux 7 et 8 , avec les mêmes résultats. C'est la raison pour laquelle ne figure dans chacun de ces tableaux que la spécification de l'effet du revenu sans terme quadratique.
} 
avec la faible mobilité associée à ce profil, mais on ne peut exclure qu'il procède aussi d'une différence de "motilité » (Kaufmann et al., 2004) de ces deux catégories, c'est-à-dire de préférences hétérogènes en matière de déplacements et d'une distribution inégale de l'accès aux infrastructures de transport et des compétences nécessaires à la mobilité.

Tableau 4

Régressions logistiques sur les probabilités d'appartenance aux trois profils de mobilité

\begin{tabular}{|c|c|c|c|}
\hline & $\begin{array}{c}\text { Profil } 1 \\
\text { Hypo-mobiles }\end{array}$ & $\begin{array}{c}\text { Profil } 2 \\
\text { Hyper-mobiles } \\
\text { à dominante automobile }\end{array}$ & $\begin{array}{c}\text { Profil } 3 \\
\text { Hyper-mobiles à dominante } \\
\text { transports en commun }\end{array}$ \\
\hline \multicolumn{4}{|l|}{ Catégories socioprofessionnelles } \\
\hline Agriculteurs & 1,2 & 0,9 & $0,5^{\star}$ \\
\hline Artisans et commerçants & 1,3 & 1,0 & 0,7 \\
\hline Chefs d'entreprises et professions libérales & 0,8 & 1,2 & 1,1 \\
\hline Cadres supérieurs & $0,7^{\star *}$ & 1,2 & 1,3 \\
\hline Professions intermédiaires & 0,9 & $1,2^{\star}$ & 1,0 \\
\hline Employés & 1,1 & 1,0 & 1,0 \\
\hline Ouvriers & $1,3^{\star \star}$ & 0,9 & 0,9 \\
\hline \multicolumn{4}{|l|}{ Étudiants et sans activités (exclu) } \\
\hline Retraités et retirés de l'emploi & 1,1 & $0,9^{\star \star}$ & 1,1 \\
\hline \multicolumn{4}{|l|}{ Taille de l'unité urbaine de résidence } \\
\hline Commune rurale & $1,5^{\star \star}$ & 1,0 & $0,5^{\star \star}$ \\
\hline$<20,000$ habitants & $1,3^{\star *}$ & 1,1 & $0,6^{\star \star}$ \\
\hline [ 20-100 000 habitants [ (exclu) & & & \\
\hline 100000 habitants < & 0,9 & $1,3^{\star \star}$ & 0,9 \\
\hline Paris & $0,5^{\star \star}$ & $0,6^{\star \star}$ & $4,1^{\star \star}$ \\
\hline \multicolumn{4}{|l|}{ Sexe } \\
\hline Femme & 1,0 & $1,1^{\star *}$ & $0,8^{\star *}$ \\
\hline Homme (exclu) & & & \\
\hline \multicolumn{4}{|l|}{ Âge } \\
\hline \multicolumn{4}{|l|}{$<30($ exclu $)$} \\
\hline [30-40 [ & $0,8^{\star \star}$ & 1,1 & 1,2 \\
\hline [40-50 [ & 1,0 & 1,1 & 0,9 \\
\hline [50-60 [ & 1,0 & 1,0 & 1,1 \\
\hline $60<$ & $1,2^{*}$ & 1,0 & $0,7^{\star *}$ \\
\hline Nombre d'unité de consommation & $0,8^{\star *}$ & $1,1^{\star \star}$ & 1,1 \\
\hline Log du revenu simulé par UC & $0,6^{* *}$ & $1,4^{\star *}$ & 1,1 \\
\hline \multicolumn{4}{|l|}{ Diplôme } \\
\hline 3ème cycle & 0,8 & 1,0 & 1,2 \\
\hline 2ème cycle & $0,5^{\star \star}$ & $1,2^{\star}$ & $1,5^{\star \star}$ \\
\hline 1er cycle & 1,0 & $1,2^{\star}$ & 0,9 \\
\hline $\mathrm{Bac}$ & 1,1 & 1,1 & 1,0 \\
\hline CAP BEP & $1,2^{\star}$ & 1,0 & 0,9 \\
\hline BEPC & $1,3^{*}$ & 0,9 & 1,0 \\
\hline \multicolumn{4}{|l|}{ CEP sans (exclu) } \\
\hline Constante & $0,3^{\star *}$ & $1,5^{\star \star}$ & $0,1^{\star \star}$ \\
\hline Effectif & 13216 & 13216 & 13216 \\
\hline
\end{tabular}

Lecture : modèles logit binomiaux. En toute rigueur, la variable dépendante étant de nature polytomique, il conviendrait d'effectuer ici un logit polytomique non ordonné. Pour simplifier la lecture des résultats, on a choisi de présenter les résultats de plusieurs logit indépendants, dont les résultats ne sont pas fondamentalement différents de ceux obtenus par un modèle polytomique quant à l'interprétation de la signification des effets estimés.

Les variables continues introduites dans les modèles présentés dans ce tableau sont standardisées (centrées sur leur moyenne ; s'agissant du revenu, la valeur moyenne est celle du log du revenu, et non celle du revenu lui-même). Pour les variables catégorielles, on utilise le codage d'effet, de sorte que la catégorie de référence pour chacune de ces variables n'est pas une catégorie spécifique, mais la valeur moyenne de la variable dépendante observée pour l'ensemble des catégories. Par exemple, un coefficient relatif à la catégorie " ouvriers » mesure la déviation de cette catégorie par rapport à la moyenne des autres catégories, à autres caractéristiques contrôlées. Pour chaque variable catégorielle, la catégorie omise dans le modèle l'est pour des raisons de multicolinéarité, et ne correspond pas à une catégorie de référence. Les effets estimés sont présentés sous forme d'odds ratio, dont les valeurs potentielles varient de 0 à $+\infty$. Une valeur égale à 1 correspond à l'absence d'effet, une valeur supérieure à 1 , à un effet positif et une valeur inférieure 1 , à un effet négatif. * : significatif au seuil de $5 \% ;{ }^{* *}$ : significatif au seuil de $1 \%$.

Champ : ménages possédant au moins un véhicule automobile, France métropolitaine.

Source : enquête nationale Transports et déplacements (ENTD) 2008, Insee. 


\section{Les liens entre les caractéristiques des véhicules et le profil de mobilité s'avèrent plus ténus}

Contrairement aux caractéristiques sociodémographiques des répondants, les propriétés des véhicules disponibles semblent relativement peu liées à la position occupée au sein de l'espace des habitudes de transports et déplacements construit par l'analyse factorielle. La projection en variables supplémentaires d'une série de variables indicatrices renseignant la possession de véhicules de plus de $6 \mathrm{cv}$ ou de moins de $6 \mathrm{cv}$, de véhicules allemandes, français ou d'autres origines, de véhicules neufs ou d'occasion ne fait ainsi pas apparaître de contrastes très prononcés dans l'espace formé par les deux premiers axes de l'ACM (cf. graphique VII $)^{8}$. La plupart des points moyens des catégories correspondantes se trouvent assez proches du centre du plan factoriel. Seuls font exception la possession et l'usage de véhicules neufs de plus de $6 \mathrm{cv}$ de marque étrangère, et plus particulièrement allemande, dont les points moyens correspondants apparaissent légèrement décalés vers le quadrant sud-ouest du plan (cf. graphique VII - B).

Cette tendance est toutefois peu accentuée, ce que confirme la distribution uniforme des différents types de véhicules au sein des trois profils de la typologie. Certes les véhicules les plus puissants (6cv fiscaux et plus) se rencontrent plus fréquemment dans le deuxième profil que dans les deux autres, surtout lorsqu'ils sont acquis neufs et, en sens inverse, les véhicules de moins de $6 \mathrm{cv}$ fiscaux, en particulier d'occasion, sont

8. Les axes suivants ne s'avèrent pas plus discriminants.

\section{Graphique VII \\ Projection des points moyens des catégories de véhicules dans le plan des deux premiers facteurs de l'ACM \\ $A$ - État d'acquisition, puissance et origine}

Axe 2

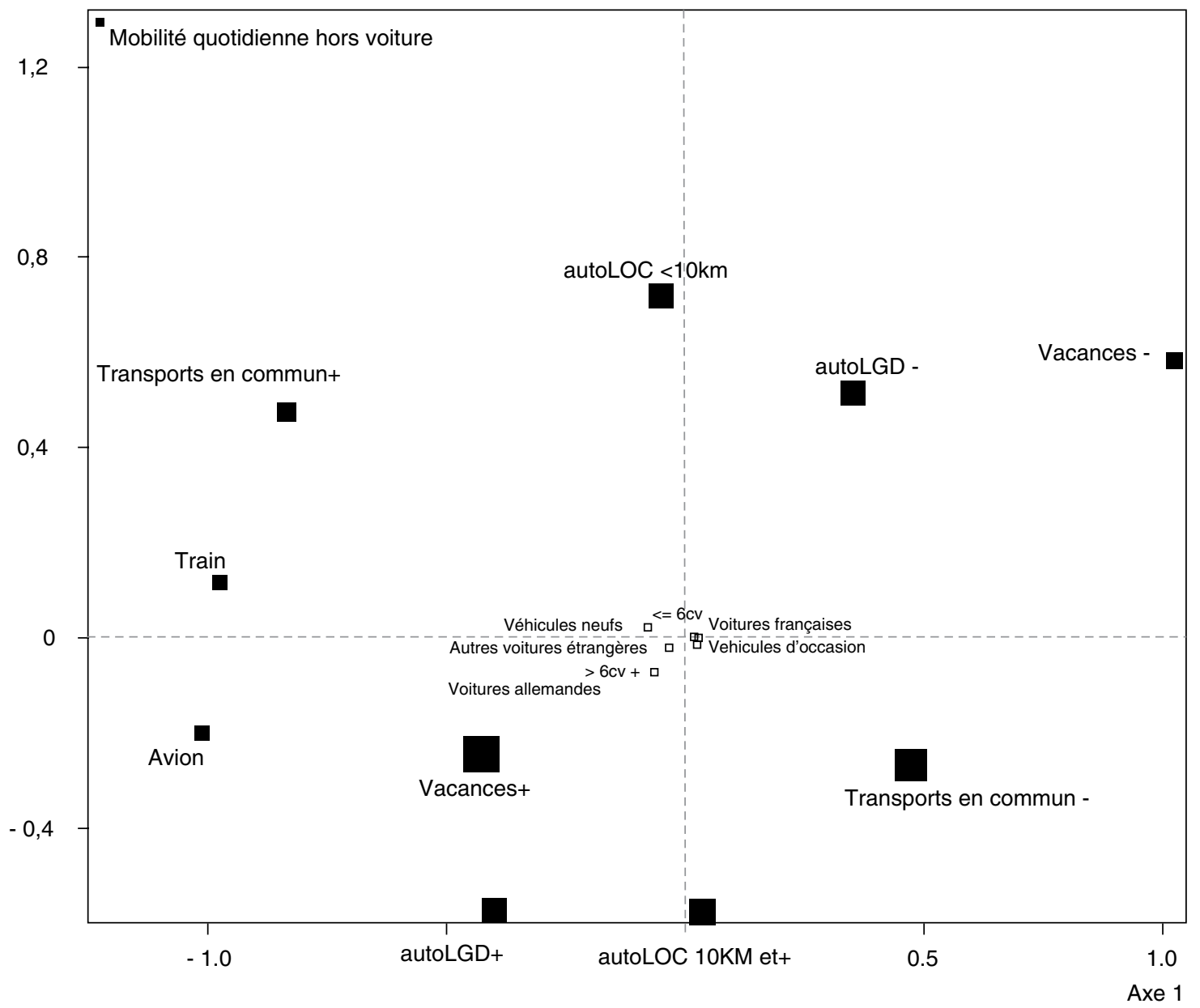


plus fréquents dans le premier (cf. tableau 5). Puissance et état d'acquisition des véhicules semblent donc liés aux habitudes de déplacement de leurs conducteurs, l'acquisition de véhicules neufs et puissants, en particulier, allant de pair avec une forte mobilité automobile, mais les écarts observés demeurent peu importants.

L'origine des véhicules (français ou étrangers) ne semble pas non plus totalement indifférente aux caractéristiques des habitudes de déplacement.
Alors que la possession de véhicules français apparaît relativement plus fréquente chez les moins mobiles (profil 1), les plus mobiles à dominante automobile (profil 2) semblent en sens inverse priser davantage les véhicules étrangers (cf. tableau 6). De nouveau, les écarts observés sont toutefois d'assez faible ampleur.

L'amplitude relativement modeste des écarts observés contraste ainsi avec celle des écarts observés au regard des caractéristiques

\section{Graphique VII (suite) \\ B - État d'acquisition, puissance et origine détaillés}

Axe 2

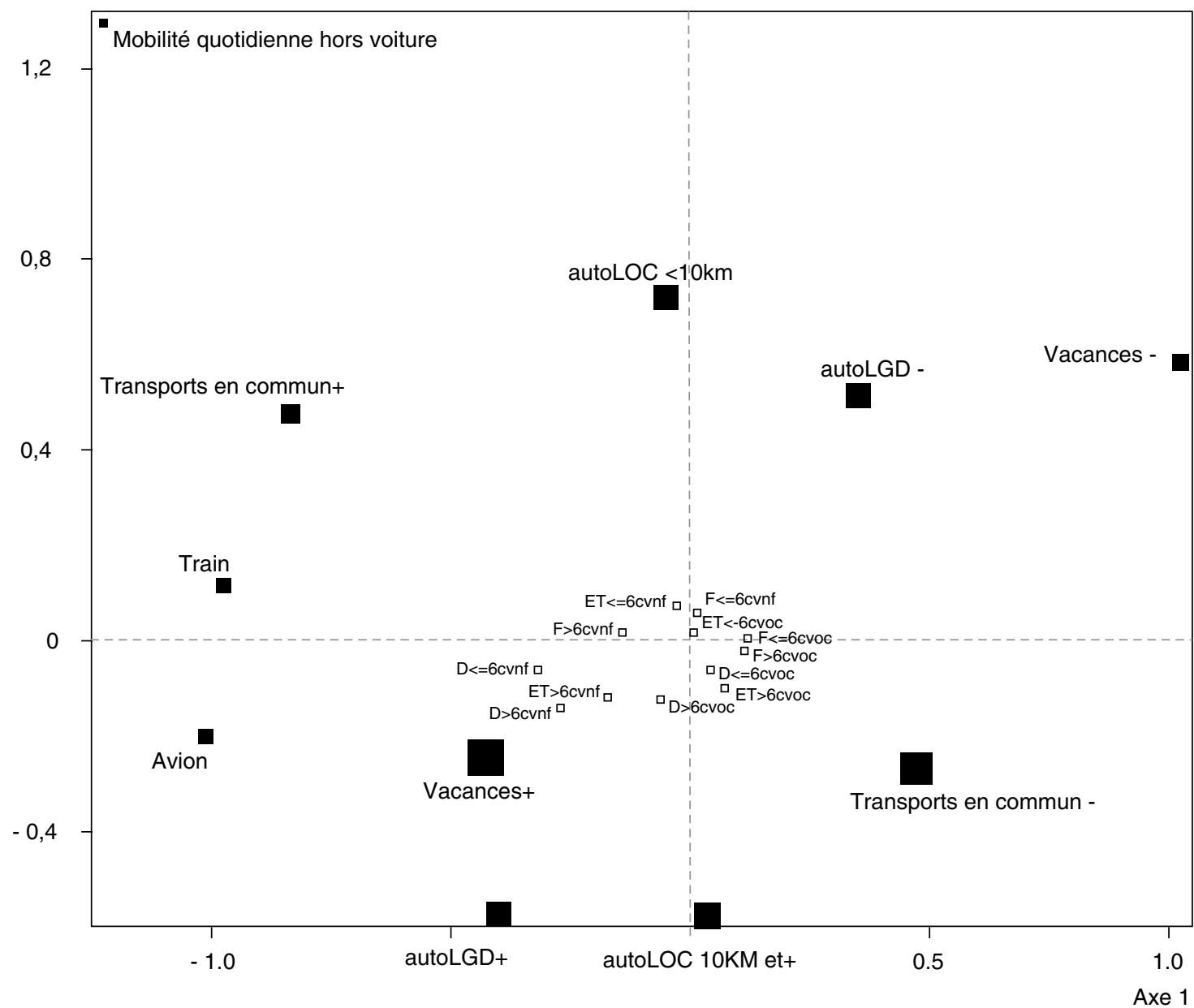

Lecture : les différentes catégories de véhicules sont désignées par les abréviations suivantes :

$F<=6 c v n f:$ Voitures françaises neuves de $6 \mathrm{cv}$ ou moins

$F<=6 \mathrm{cvoc}$ : Voitures françaises d'occasion de $6 \mathrm{cv}$ ou moins

F>6cvnf: Voitures françaises neuves de plus de $6 \mathrm{cv}$

F>6cvoc: Voitures françaises d'occasion de plus de $6 \mathrm{cv}$

$D<=6 \mathrm{cvnf}$ : Voitures allemandes neuves de $6 \mathrm{cv}$ ou moins

$D<=6 \mathrm{cvoc}$ : Voitures allemandes d'occasion de $6 \mathrm{cv}$ ou moins

$D>6 c v n f$ : Voitures allemandes neuves de plus de $6 \mathrm{cv}$

$D>6 \mathrm{cvoc}$ : Voitures allemandes d'occasion de plus de $6 \mathrm{cv}$

$E T<=6 \mathrm{cvnf}$ : Voitures étrangères autres qu'allemandes neuves de $6 \mathrm{cv}$ ou moins

$E T<=6 \mathrm{cvoc}$ : Voitures étrangères autres qu'allemandes d'occasion de $6 \mathrm{cv}$ ou moins

ET>6cvnf: Voitures étrangères autres qu'allemandes neuves de plus de $6 \mathrm{cv}$

$E T>6 \mathrm{cvoc}$ : Voitures étrangères autres qu'allemandes d'occasion de plus de $6 \mathrm{cv}$ :

Les carrés représentant les modalités des variables actives ont une taille proportionnelle à la taille relative des effectifs de ces modalités. Champ : ménages possédant au moins un véhicule automobile, France métropolitaine.

Source : enquête nationale Transports et déplacements (ENTD) 2008, Insee. 
sociodémographiques des ménages s'agissant de la puissance, de l'état d'acquisition et de l'origine des véhicules (cf. graphique IV et tableaux 1 et 2). Il reste que la signification de ces écarts, notamment des écarts portant sur la catégorie socioprofessionnelle des personnes de référence des ménages, n'est pas directement interprétable. La multimotorisation croissante des ménages et le fait que l'utilisation des véhicules tende à s'y individualiser invite à raisonner davantage sur les conducteurs que sur les détenteurs des véhicules. D'autre part, ces écarts mêlent des effets de revenu et des effets associés aux caractéristiques des habitudes de déplacement plus directement liées aux conditions d'emploi, de localisation et de logement, notamment, qu'il convient de démêler de ce qui relève davantage d'effets propres aux styles de vie des différentes groupes sociaux et des formes de distinction ou de compétition statutaire qui se nouent dans les caractéristiques de leur équipement automobile. Une mesure plus précise de ces effets et de la manière dont ils s'articulent peut être obtenue au moyen de régressions logistiques des probabilités de détention des différentes catégories de véhicules sur un ensemble de caractéristiques sociodémographiques, pour la population prise dans son ensemble, tout d'abord, puis pour chacun des profils issus de la typologie des habitudes de déplacement pris séparément.

\section{L'automobile : un bien qui demeure « classant »}

D our établir la part de ce que les caractéristiques de l'équipement automobile doivent aux contraintes fonctionnelles et aux incitations associées aux habitudes de transport et de ce qu'elles doivent à l'identité des groupes sociaux appréhendée ici par la catégorie socioprofessionnelle des conducteurs, on se propose de régresser la probabilité de disposer de différentes catégories de véhicules au regard des caractéristiques de puissance, d'état d'acquisition et d'origine sur un ensemble de caractéristiques sociodémographiques dont on entend distinguer l'effet propre. On utilise à cet effet sept régressions logistiques : restreintes aux individus usant d'au moins un véhicule automobile au sein de leur ménage, elles régressent, sur les mêmes variables que précédemment, sept variables à

Tableau 5

Puissance et état d'acquisition des véhicules selon le profil de mobilité

\begin{tabular}{|l|c|c|c|c|}
\hline & $\begin{array}{c}\text { Profil 1 } \\
\text { Hypo-mobiles }\end{array}$ & $\begin{array}{c}\text { Profil 2 Hyper-mobiles } \\
\text { à dominante automobile }\end{array}$ & $\begin{array}{c}\text { Profil 3 Hyper-mobiles à dominante } \\
\text { transports en commun }\end{array}$ & Ensemble \\
\hline $\begin{array}{c}\text { Véhicules neufs } \\
\text { 6cv et plus }\end{array}$ & 8,1 & 13,0 & 11,3 & 20,4 \\
Moins de 6cv & 21,2 & 20,9 & 21,0 \\
\hline $\begin{array}{l}\text { Véhicules d'occasion } \\
\text { 6cv et plus }\end{array}$ & 27,5 & 31,7 & 28,0 & 30,1 \\
Moins de 6cv & 55,5 & 51,1 & 48,0 & 52,0 \\
\hline
\end{tabular}

Lecture : 8,1 \% des ménages hypo-mobiles disposent d'au moins un véhicule neuf de 6cv et plus.

Champ : ménages possédant au moins un véhicule automobile, France métropolitaine.

Source : enquête nationale Transports et déplacements (ENTD) 2008, Insee.

Tableau 6

Origine des véhicules selon le profil de mobilité

\begin{tabular}{|c|c|c|c|c|}
\hline & $\begin{array}{c}\text { Profil } 1 \\
\text { Hypo-mobiles }\end{array}$ & $\begin{array}{l}\text { Profil } 2 \\
\text { Hyper-mobiles } \\
\text { à dominante } \\
\text { automobile }\end{array}$ & $\begin{array}{c}\text { Profil } 3 \\
\text { Hyper-mobiles } \\
\text { à dominante transports } \\
\text { en commun }\end{array}$ & Ensemble \\
\hline Véhicules de marque française & 71,1 & 68,1 & 66,2 & 68,8 \\
\hline Véhicules de marque allemande & 11,4 & 15,3 & 13,0 & 14,0 \\
\hline Véhicules de marque étrangère (autre qu'allemande) & 26,8 & 28,1 & 27,6 & 27,7 \\
\hline
\end{tabular}

Lecture : $71,1 \%$ des ménages hypo-mobiles disposent d'au moins un véhicule de marque française.

Champ : ménages possédant au moins un véhicule automobile, France métropolitaine.

Source : enquête nationale Transports et déplacements (ENTD) 2008, Insee. 
deux modalités ${ }^{9}$, qui permettent de comparer la possession de tout autre type de véhicule à la possession d'un véhicule d'origine française (1), d'origine allemande (2), d'origine étrangère autre qu'allemande (3), à l'état neuf de plus de $6 \mathrm{cv}$ (4), à l'état neuf de $6 \mathrm{cv}$ ou moins (5), en seconde main de plus de $6 \mathrm{cv}(6)$, et en seconde main de $6 \mathrm{cv}$ ou moins (7) (cf. tableau 7).

9. Des analyses antérieures basées sur une classification des marques plus détaillée ont démontré que dans l'ENTD 2008 les principales distinctions se résument efficacement à la typologie agrégée qui fait l'objet de l'analyse présentée ici.

\section{Tableau 7}

Régressions logistiques sur les probabilités de possession de véhicules selon l'origine, l'état d'acquisition et la puissance fiscale

\begin{tabular}{|c|c|c|c|c|c|c|c|}
\hline & Française & Allemande & Étrangère & $\begin{array}{l}\text { Neuf } \\
>6 \mathrm{cv}\end{array}$ & $\begin{array}{l}\text { Neuf } \\
<=6 \mathrm{cv}\end{array}$ & $\begin{array}{l}\text { Occasion } \\
>6 c v\end{array}$ & $\begin{array}{c}\text { Occasion } \\
<=6 \mathrm{v}\end{array}$ \\
\hline \multicolumn{8}{|l|}{ Catégorie socioprofessionnelle } \\
\hline Agriculteurs & $1,4^{*}$ & 0,8 & 0,7 & 1,3 & 1,0 & 1,2 & 1,1 \\
\hline Artisans et commerçants & 0,8 & $1,6^{\star *}$ & $1,5^{\star}$ & $1,6^{\star \star}$ & 0,8 & $1,4^{\star *}$ & 1,0 \\
\hline $\begin{array}{l}\text { Chefs d'entreprises et professions } \\
\text { libérales }\end{array}$ & $0,4^{* *}$ & $2,1^{\star *}$ & $1,7^{*}$ & 1,3 & 0,8 & $1,5^{\star}$ & 0,8 \\
\hline Cadres supérieurs & $1,3^{\star \star}$ & $0,7^{\star \star}$ & 1,0 & 0,9 & 1,0 & 0,8 & 1,1 \\
\hline Professions intermédiaires & 1,1 & $0,8^{\star *}$ & 1,0 & 0,9 & 1,1 & 0,9 & 1,1 \\
\hline Employés & 1,1 & $0,7^{\star *}$ & 0,9 & 1,0 & 1,1 & $0,8^{\star *}$ & 1,0 \\
\hline $\begin{array}{l}\text { Ouvriers } \\
\text { Étudiants et sans activités (exclu) }\end{array}$ & 1,1 & 0,8 & 0,9 & $0,6^{\star \star}$ & 1,0 & $0,8^{\star *}$ & 1,1 \\
\hline Retraités et retirés de l'emploi & 1,0 & 1,1 & 1,0 & 1,0 & 1,0 & 1,1 & $0,9^{*}$ \\
\hline \multicolumn{8}{|l|}{ Taille de l'unité urbaine de résidence } \\
\hline $\begin{array}{l}\text { Commune rurale } \\
<20000 \text { habitants }\end{array}$ & $\begin{array}{l}1,1 \\
1,1\end{array}$ & $\begin{array}{l}1,0 \\
0,9\end{array}$ & $\begin{array}{l}1,1 \\
0,9\end{array}$ & $\begin{array}{l}0,9 \\
1,0\end{array}$ & 0,9 & $\begin{array}{l}1,1 \\
1,0\end{array}$ & $\begin{array}{l}1,2 \\
1,0\end{array}$ \\
\hline [ 20000 - 100000 habitants [ (exclu) & & & & & & & \\
\hline 100000 habitants $<$ & $0,9^{*}$ & 1,2 & 1,0 & 1,1 & 0,9 & 1,0 & 0,9 \\
\hline Paris & 1,0 & $0,8^{\star \star}$ & 0,9 & 1,1 & $1,2^{*}$ & $0,9^{*}$ & $0,8^{\star \star}$ \\
\hline \multicolumn{8}{|l|}{ Sexe } \\
\hline $\begin{array}{l}\text { Femme } \\
\text { Homme (exclu) }\end{array}$ & 1,0 & 1,0 & 1,0 & $0,8^{\star *}$ & 1,0 & 1,0 & 1,0 \\
\hline \multicolumn{8}{|l|}{ Âge } \\
\hline$<30$ (exclu) & & & & & & & \\
\hline$[30-40[$ & $0,8^{\star *}$ & $1,4^{\star *}$ & $1,2^{*}$ & 1,0 & $0,8^{\star *}$ & $1,4^{\star *}$ & 1,0 \\
\hline$[40-50[$ & $0,9^{\star}$ & 1,1 & 1,0 & 1,2 & 1,0 & 1,1 & 0,9 \\
\hline$[50-60[$ & 1,1 & 0,9 & 1,1 & $1,7^{\star}$ & $1,4^{\star \star}$ & 1,0 & 0,9 \\
\hline $60<$ & $1,4^{\star \star}$ & $0,6^{\star \star}$ & 0,8 & $2,0^{\star \star}$ & $2,2^{\star \star}$ & $0,7^{\star \star}$ & $0,6^{\star \star}$ \\
\hline Nombre d'unité de consommation & 1,0 & 1,1 & $1,3^{\star \star}$ & 1,1 & $0,8^{\star \star}$ & $1,3^{\star \star}$ & $1,1^{*}$ \\
\hline Log du revenu simulé par UC & $0,9^{\star \star}$ & $1,5^{\star \star}$ & $1,2^{\star \star}$ & $1,5^{\star \star}$ & 1,0 & $1,2^{\star \star}$ & $0,9^{\star \star}$ \\
\hline \multicolumn{8}{|l|}{ Diplôme } \\
\hline 3ème cycle & 1,1 & 1,0 & $0,7^{*}$ & 0,9 & 1,2 & 0,8 & 1,1 \\
\hline 2ème cycle & 1,1 & 1,0 & 1,0 & 1,0 & 1,1 & 0,8 & $1,2^{*}$ \\
\hline 1er cycle & 1,0 & 1,1 & 1,1 & 1,2 & 1,2 & 0,9 & 1,0 \\
\hline $\mathrm{Bac}$ & 0,9 & 0,9 & $1,3^{*}$ & 1,0 & 1,0 & 1,1 & 1,0 \\
\hline CAP BEP & 1,0 & 1,1 & 1,1 & 0,9 & $0,8^{* *}$ & 1,1 & $1,1^{*}$ \\
\hline \multicolumn{8}{|l|}{ CEP sans (exclu) } \\
\hline Constante & $2,9^{\star \star}$ & $0,2^{* *}$ & $0,2^{* *}$ & $0,1^{\star *}$ & $0,2^{* *}$ & $0,5^{\star \star}$ & 0,9 \\
\hline Log-Likelihood Ratio Chi-squared & 0 & $389^{\star *}$ & $282^{\star \star}$ & $854^{\star \star}$ & $735^{\star \star}$ & $493^{\star \star}$ & $779^{\star \star}$ \\
\hline Bayesian Information Criterion & -23 & -152 & -45 & -617 & -498 & -256 & -542 \\
\hline Effectif & 13216 & 13216 & 13216 & 13216 & 13216 & 13216 & 13216 \\
\hline
\end{tabular}

Lecture : modèles logit binomiaux. Les variables continues introduites dans les modèles présentés dans le tableau 7 sont standardisées (centrées sur leur moyenne ; s'agissant du revenu, la valeur moyenne est celle du log du revenu, et non celle du revenu lui-même). Pour les variables catégorielles, on utilise le codage d'effet, de sorte que la catégorie de référence pour chacune de ces variables n'est pas une catégorie spécifique, mais la valeur moyenne de la variable dépendante observée pour l'ensemble des catégories. Par exemple, un coefficient relatif à la catégorie " ouvriers » mesure la déviation de cette catégorie par rapport à la moyenne des autres catégories, à autres caractéristiques contrôlées. Pour chaque variable catégorielle, la catégorie omise dans le modèle l'est pour des raisons de multicolinéarité, et ne correspond pas à une catégorie de référence. Les effets estimés sont présentés sous forme d'odds ratio, dont les valeurs potentielles varient de 0 à $+\infty$. Une valeur égale à 1 correspond à l'absence d'effet, une valeur supérieure à 1, à un effet positif et une valeur inférieure 1 , à un effet négatif. * : significatif au seuil de $5 \%$; ${ }^{*}$ : significatif au seuil de $1 \%$.

Champ : ménages possédant au moins un véhicule automobile, France métropolitaine.

Source : enquête nationale Transports et déplacements (ENTD) 2008, Insee. 
Les rapports entre puissance automobile, marque et catégorie socioprofessionnelle confirment le rôle de marqueur social de la voiture

Le contrôle des effets associés à la catégorie socioprofessionnelle par les variables de sexe, d'âge, de diplôme, de statut d'activité professionnelle, ainsi que de lieu de résidence, de taille et de niveau de ressources économiques du ménage d'appartenance, permet d'isoler ce qui, dans les caractéristiques des automobiles dont disposent les conducteurs, relève en propre de leur appartenance socioprofessionnelle.
À cette fin, les effets relatifs à la catégorie socioprofessionnelle, exprimés sous la forme d'odds ratios, sont représentés graphiquement (cf. graphique VIII).

Les effets de la catégorie socioprofessionnelle estimés par ces modèles logistiques confirment assez largement l'hypothèse selon laquelle l'automobile demeurerait aujourd'hui en France un marqueur de la position et de catégorie sociale. Des différences très larges et statistiquement significatives apparaissent entre les catégories socioprofessionnelles pour une bonne part des sept catégories de véhicules

\section{Graphique VIII}

L'effet de la csp sur la distribution des différents types de véhicules

\section{A - Selon l'origine du véhicule}

Selon l'origine du véhicule

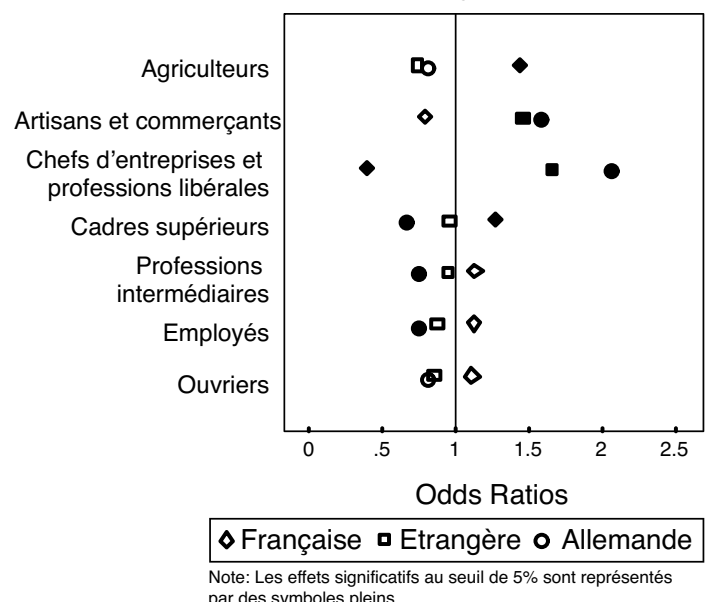

B - Selon la puissance fiscale (véhicule neuf) Véhicule neuf selon la puissance fiscale

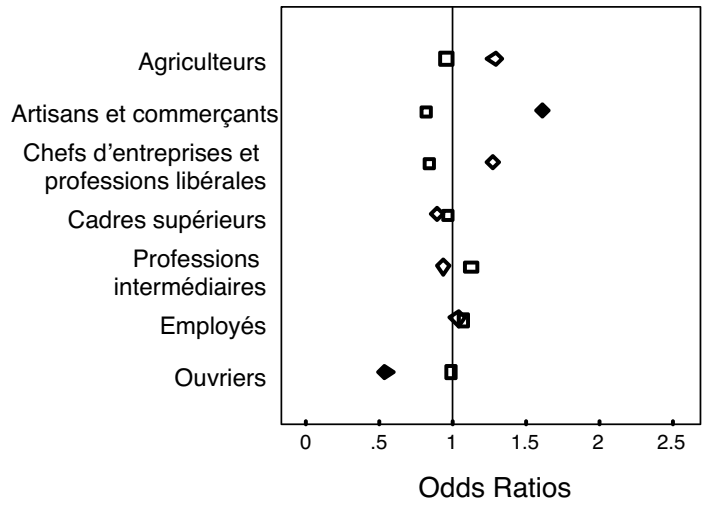

$\Delta>6$ chevaux $\square<=6$ chevaux Note: Les effets significatifs au seuil de $5 \%$ ont représentés par des symboles pleins

\section{C - Selon la puissance fiscale (véhicule d'occasion)}

Vehicules d'occasion selon la puissance fiscale

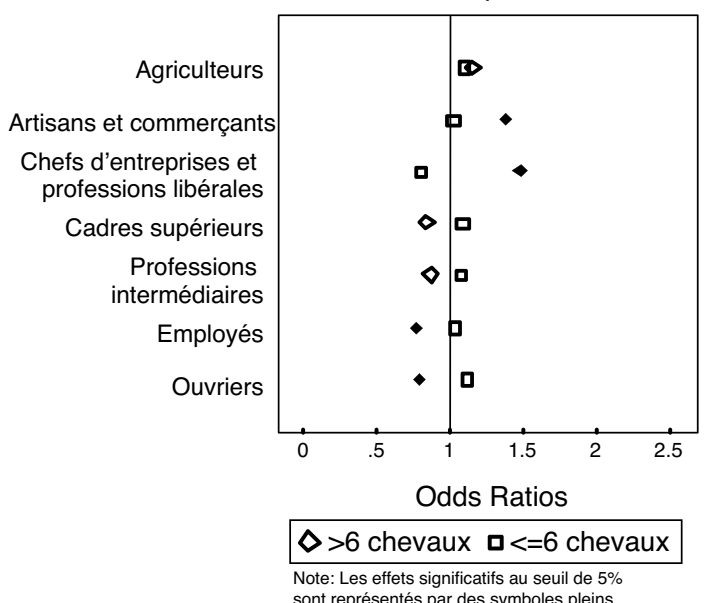

Lecture : modèles logit binomiaux (cf. tableau 7). Sont représentés, sous forme d'odds ratios, les effets de la catégorie socioprofessionnelle des répondants sur la probabilité d'un ménage de disposer d'au moins d'une automobile selon l'origine du véhicule (A), la puissance fiscale à l'état neuf $(B)$ et en seconde main $(C)$. Exemple : Les répondants appartenant à la catégorie socioprofessionnelle des chefs d'entreprises et professions libérales ont deux fois plus de chance de disposer d'un véhicule d'origine allemande que la moyenne indépendamment du sexe, de l'âge, du diplôme, du statut d'activité professionnelle, ainsi que du lieu de résidence, de la taille et des ressources économiques du ménage d'appartenance (effets de contrôle non représentés). Les effets significatifs au seuil de 5 \% sont représentés par des symboles pleins.

Champ : ménages possédant au moins un véhicule automobile, France métropolitaine.

Source : enquête nationale Transports et déplacements (ENTD) 2008, Insee. 
précédemment définies, et ces différences résistent au contrôle par les autres variables introduites dans l'analyse.

Dans le détail, on peut tout d'abord relever l'effet de catégorie sociale attaché à l'origine nationale des véhicules. L'attrait pour les marques françaises apparait, de manière statistiquement significative, comme un marqueur de la catégorie des agriculteurs et dans une moindre mesure de celle des cadres supérieurs. Il est également un marqueur de la catégorie des chefs d'entreprises et des professions libérales, mais dans le sens inverse : ceux-ci ont plus de deux fois moins de chances que les autres catégories socioprofessionnelles de conduire des véhicules français. Ce goût pour les marques étrangères est partagé par les artisans et les commerçants, et on observe un effet particulier pour les véhicules de marque allemande dont la possession départage les travailleurs indépendants et les autres catégories socioprofessionnelles.

S'agissant de l'état d'acquisition et de la puissance des véhicules, les écarts entre les catégories socioprofessionnelles sont moins prononcés et surtout rarement significatifs. On distingue néanmoins une inclination des travailleurs indépendants pour les véhicules puissants. Cette prédilection semble jouer indépendamment de l'état d'acquisition, mais elle n'est statistiquement significative, dans le cas des véhicules neufs, que pour les artisans et commerçants alors qu'elle l'est plus largement dans le cas des véhicules d'occasion, pour lesquels elle concerne aussi les chefs d'entreprises et professions libérales. En sens inverse, les ouvriers, dans le cas des véhicules neufs, auxquels s'ajoutent les employés, dans le cas des véhicules d'occasion, se distinguent par une moindre propension à disposer de véhicules de cette puissance. Il faut avoir ici présent à l'esprit ce que les associations entre catégories sociales et types de véhicules peuvent devoir aux phénomènes de multi-motorisation, selon le « rang » occupé parmi les véhicules du ménage. On peut penser, à cet égard, que les véhicules de petite cylindrée ont davantage de chances, en cas de multi-motorisation du ménage, d'entrer dans la catégorie des véhicules de " second rang ", ce qui rend inévitablement l'interprétation des effets mis en évidence plus délicate.

Les effets associés à la catégorie socioprofessionnelle des conducteurs peuvent s'interpréter comme résultant des spécificités et des exigences fonctionnelles de l'activité exercée, l'attrait pour les véhicules les plus puissants traduisant par exemple l'effet d'habitudes de déplacement différenciées par les contraintes propres aux différents groupes socioprofessionnels. Cette interprétation est toutefois fortement affaiblie par la relative stabilité des effets mis en évidence lorsque la régression des probabilités de disposer de véhicules de différents types est répliquée séparément sur les conducteurs des trois profils identifiés précédemment à partir de la caractérisation de leurs habitudes de déplacement (cf. tableau 8). La similitude de l'effet associé à la catégorie des artisans et commerçants s'agissant de l'attrait pour les véhicules les plus puissants au sein du premier profil (personnes les moins mobiles), du deuxième (où les déplacements automobiles sont les plus nombreux et les plus fréquents) et, plus encore, son renforcement au sein du troisième profil (personnes dont la mobilité élevée est la moins dépendante de l'automobile), ne permet en particulier pas de réduire cet effet à celui des habitudes de déplacement propres aux différents groupes professionnels.

L'impact de la variable catégorie socioprofessionnelle renvoie donc sans doute plus largement à des effets de socialisation ancrés dans l'identité et le style de vie des différents groupes sociaux, qui se forgent et se transmettent vraisemblablement dans l'environnement de travail, mais aussi dans l'environnement social et familial. Le clivage prononcé entre indépendants et salariés s'agissant de l'attrait pour les véhicules de marque étrangère, et singulièrement allemande, peut sans doute s'interpréter en ces termes (cf. tableau 7), et confirme des tendances observées de longue date (Choquet, 1983).

Ce type d'interprétations, qui possède une forte affinité avec les analyses en termes d'homologie (Bourdieu, 1979), en ce qu'il met en relation l'espace des propriétés sociales des conducteurs, structuré par des oppositions de type indépendant/salarié, privé/public, et l'espace des propriétés des automobiles, se voit parfois opposer des interprétations en termes de compétition statutaire, qui évoquent davantage les effets d'imitation ou de consommation ostentatoire (Veblen, 1899). Ces interprétations rencontrent un certain écho dans les critiques adressées par David Gartman aux thèses de Bourdieu (Gartman, 2004). Dans le contexte de standardisation de la production en grande série caractéristique de l'industrie automobile d'après-guerre, la voiture particulière perdrait l'essentiel de ses propriétés distinctives, au sens de la distinction entre des sous-cultures et des styles de vie cohérents, ancrés dans des habitus 


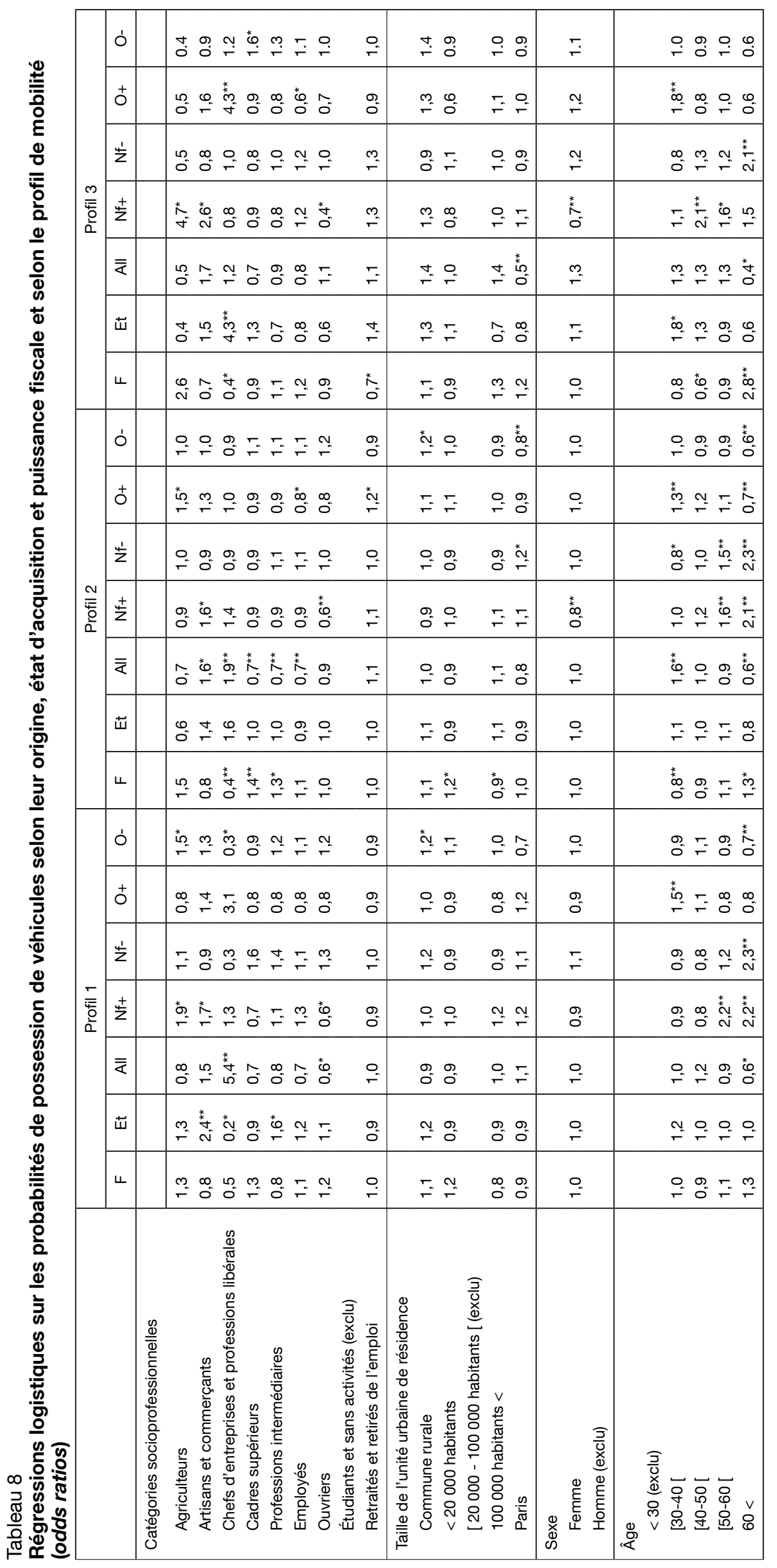




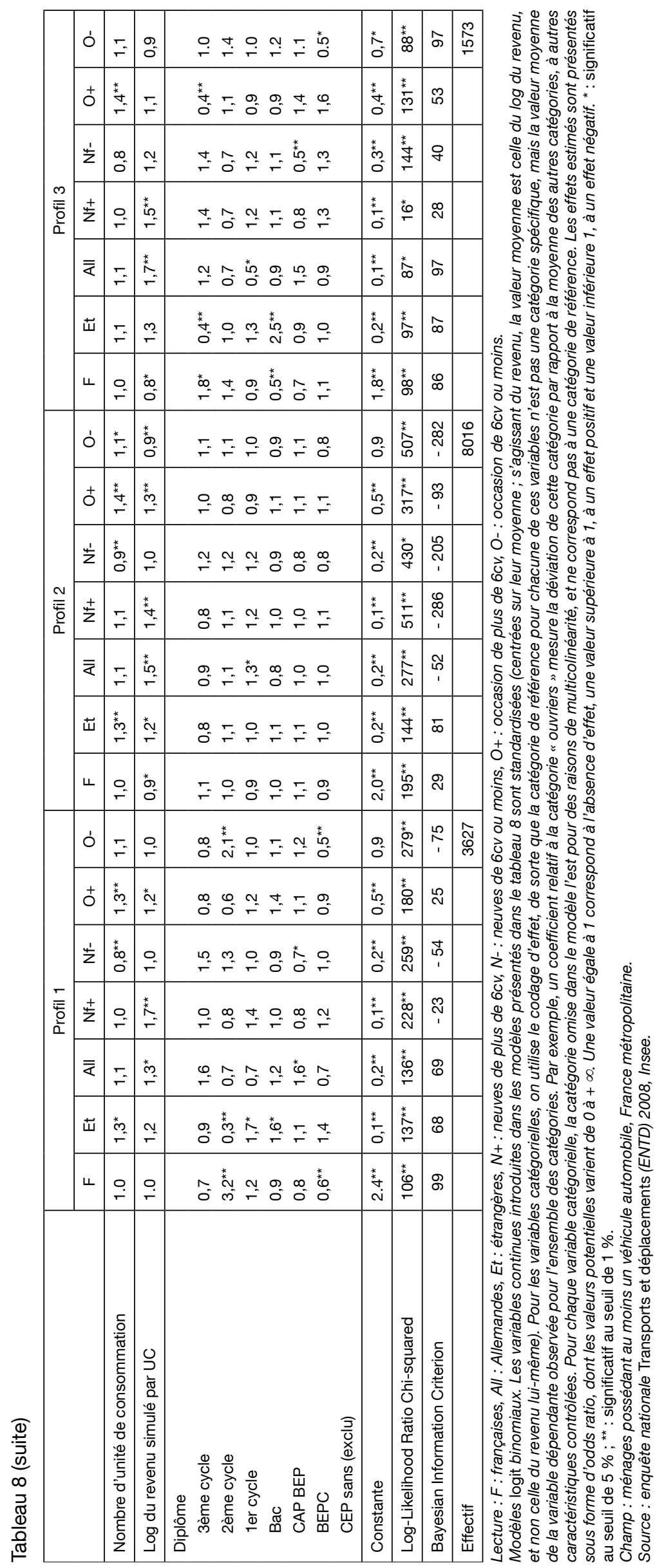


" de classe », et répondrait davantage à une compétition statutaire indexée sur des critères homogènes d'un groupe social à l'autre. Les écarts observés dans les analyses de régressions des tableaux 7 et 8 quant à la puissance des véhicules et quant aux marques - telles qu'elles sont saisies ici par la distinction seulement entre marques françaises et étrangères - renverraient ainsi à un ordonnancement hiérarchique des groupes sociaux faisant totale abstraction de l'échelle des revenus.

Cette dernière interprétation ne semble toutefois pas pleinement justifiée par les résultats. Bien que la nomenclature française des catégories socioprofessionnelles ne comporte pas de dimension hiérarchique explicite et unidimensionnelle, les effets de sens contraire observés pour les chefs d'entreprises et professions libérales d'un côté, et les cadres supérieurs, de l'autre, s'agissant en particulier de l'attrait pour les véhicules de marque française et allemande ne cadrent pas avec la position relative de ces catégories dans les échelles de statut ou de prestige construites sur données françaises (Chambaz et al., 1998 ; Cousteaux et Lemel, 2004 ; Lemel, 2006). Les effets observés au niveau de la puissance des véhicules peuvent apparaître davantage en conformité avec ce type d'échelles, mais, de nouveau, les inversions de signe des effets observées au sommet de la hiérarchie suggère plutôt la prégnance de clivages catégoriels du type de celui opposant les salariés aux indépendants.

\section{La force des effets de l'âge et du revenu confirme le caractère de "bien supérieur " des grosses cylindrées achetées à l'état neuf...}

Les résultats des sept régressions logistiques présentées dans le tableau 7 font aussi ressortir l'effet d'autres variables. En premier lieu, disposer de véhicules neufs, quelle qu'en soit la puissance, est significativement plus probable chez les conducteurs de plus de 50 ans, et plus encore, de plus de 60 ans. Cet effet de l'âge est cohérent avec ce que l'on sait par ailleurs du recul de l'âge moyen au premier achat de véhicule neuf aujourd'hui en France ${ }^{10}$. En sens inverse, la probabilité de disposer de véhicules d'occasion décroît au-dessus de 50 ans et, pour les plus puissants, est plus grande chez les 30-40 ans. L'équipement automobile semble ainsi soumis à une forte différenciation en fonction de l'âge, dont il est difficile de déterminer, sur la base de données transversales, si celle-ci relève davantage d'un effet de génération que d'un effet de cycle de vie. L'interprétation générationnelle, elle aussi cohérente avec le recul observé de l'âge moyen des acquéreurs de véhicules neufs, conduit à relier les positions opposées des trentenaires, d'une part, et des quinquagénaires et sexagénaires, d'autre part, à un accès encore aujourd'hui privilégié des cohortes du baby-boom à un bien particulièrement emblématique des décennies d'expansion de l'après-guerre, par contraste avec les obstacles rencontrés par les générations ultérieures dans l'accès à ces mêmes biens. L'interprétation en termes de cycle de vie suppose, quant à elle, une propension intrinsèque à disposer de véhicules neufs à mesure de l'avancée en âge, qui peut notamment évoquer une aversion plus prononcée aux risques associés à la détention de véhicules de seconde main.

Disposer de véhicules de plus de $6 \mathrm{cv}$ fiscaux, neufs ou d'occasion, est aussi fort logiquement positivement lié au niveau de revenu, exprimé, dans chacune des régressions, par le logarithme du revenu disponible du ménage par unité de consommation. Dans la mesure où les oddsratio portent sur la variation d'une unité du logarithme du revenu, les effets associés sont extrêmement prononcés, davantage pour les achats de véhicules neufs que pour les achats de véhicules d'occasion, toutefois. Il est par ailleurs remarquable que cet effet du revenu apparaisse, pour les véhicules neufs, relativement plus accentué chez les conducteurs relevant du profil le moins mobile (classe 1) que dans les deux autres profils (cf. tableau 8). Autrement dit, moins le fait de disposer d'un véhicule neuf et puissant se plie aux nécessités associées aux habitudes de déplacement, plus le revenu en est à lui seul un bon prédicteur : les caractéristiques de tels véhicules ne répondent ainsi pas véritablement aux contraintes fonctionnelles auxquelles leurs conducteurs sont ordinairement exposés. De la sorte, le caractère de « bien supérieur » de ces automobiles semble affirmé. Le fait que cet effet du revenu n'apparaisse pas significatif pour les véhicules de puissance moindre achetés neuf et qu'il s'inverse pour les véhicules d'occasion, dont l'acquisition est d'autant plus probable que le revenu est faible, vient renforcer cette interprétation.

10. 51 ans en 2009, selon les chiffres de l'Observatoire Cetelem de l'Automobile. 


\section{...mais elles perdent ce caractères quand elles sont achetées d'occasion}

Les résultats des régressions logistiques font aussi apparaître un effet de la taille du ménage, dont l'interprétation est assez incertaine. Disposer d'au moins un véhicule de $6 \mathrm{cv}$ ou plus est ainsi d'autant plus fréquent que la taille des ménages est élevée, mais ce seulement lorsqu'il s'agit de véhicules d'occasion (cf. tableau 7). Mise en regard de l'effet de revenu associé à la disposition des mêmes types de véhicules acquis neufs, cet effet de la taille du ménage suggère qu'en transitant par le marché de l'occasion, les véhicules les plus puissants non seulement perdent leur caractère de bien supérieur mais qu'ils acquièrent aussi un statut utilitaire de voiture familiale qu'ils n'avaient pas à l'état neuf.

En sens inverse, la probabilité de disposer de véhicules de puissance plus faible (moins de $6 \mathrm{cv}$ fiscaux) est négativement corrélée avec la taille du ménage, lorsqu'il s'agit de véhicules neufs, et positivement, quoique plus légèrement, lorsqu'il s'agit de véhicules d'occasion. Si la première de ces deux corrélations est cohérente avec la fonctionnalité d'autant plus faible de ce type d'équipement automobile que la taille du ménage est élevée, la seconde se comprend aisément dans la mesure où les achats de « petites » voitures de seconde main renvoient à un marché de la deuxième voiture au sein de ménages nombreux et en conséquence plus susceptibles d'être multimotorisés.

Les effets associés au lieu de résidence, enfin, sont en revanche moins prononcés, moins systématiques et de ce fait plus difficiles à l'interpréter que les précédents. Il en va de même des effets associés au diplôme, au statut d'activité professionnelle et au sexe, au-delà du fait que les femmes ont une propension significativement plus faible que les hommes à la conduite de véhicules neufs de grande cylindrée.

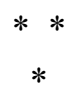

L'analyse conjointe des caractéristiques d'équipement et des habitudes de déplacement fait ressortir, en matière d'équipement automobile, des clivages entre les groupes sociaux de même nature que ceux qui s'observent plus généralement dans les styles de vie ou les habitudes de consommation. Dans un contexte de relative banalisation de l'accès à la motorisation, le statut d'acquisition des véhicules apparaît comme un marqueur social soumis à un cycle de diffusion et de banalisation que révèle l'accès socialement différencié au marché du neuf et de l'occasion. Il se combine au type de véhicule. Les voitures allemandes, en particulier les plus puissantes, apparaissent ainsi comme un marqueur spécifique de l'appartenance aux classes supérieures, en particulier chez les indépendants.

L'interprétation de ces effets demeure toutefois incertaine. Il est en particulier difficile de départager ce qui, dans les différences observées entre les groupes sociaux, relève de comportements stratégiques du type de ceux habituellement associés aux phénomènes de consommation ostentatoire (Veblen, 1970 [1899]), de ce qui renvoie davantage aux formes spécifiques de socialisation et aux « habitus » propres aux différents groupes sociaux (Bourdieu, 1979).

Malgré la subsistance d'écarts importants entre groupes sociaux quant à l'accès à la motorisation et à la distribution des différentes catégories de véhicules, le choix automobile s'avère également tributaire des contraintes fonctionnelles associées aux habitudes de déplacement. Il ne saurait donc être réduit à ses dimensions symboliques. Les caractéristiques de l'équipement automobile dépendent ainsi tout particulièrement de la taille, du revenu et du lieu de résidence des ménages. Elles sont en revanche beaucoup moins sensibles aux profils de mobilités des individus. Ici comme précédemment s'agissant de départager les comportements stratégiques de l'effet de dispositions et de traits culturels incorporés, le recours à des données longitudinales retraçant les évolutions de l'équipement automobile des ménages permettrait de mesurer plus sûrement le poids de ces différents facteurs et la portée des interprétations alternatives qui leur sont associées. 


\section{BIBLIOGRAPHIE}

Baron J.-F. (2002), « La consommation automobile depuis quarante ans », Insee Première, n844, mai.

Bell D.A. (1973). The Coming of Post-Industrial Society: A Venture in Social Forecasting, Basic Books, New York.

Besnard P. et Desplanques G. (1999), « Les catégories socioprofessionnelles à l'épreuve de la stratification temporelle des goûts », Revue Française de Sociologie, vol. 40, n¹, pp. 97-109.

Boltanski L. (1975), « Les usages sociaux de l'automobile : concurrence pour l'espace et accidents », Actes de la Recherche en Sciences Sociales, vol. $1, \mathrm{n}^{\circ} 2$, pp. 25-29.

Boltanski L. (1976), « L'encombrement et la maîtrise des biens « sans maître », Actes de la Recherche en Sciences Sociales, vol. 2, n 1, pp. 102-109.

Bourdieu P. (1979), La Distinction. Critique sociale du jugement, Éditions de Minuit, Paris.

Choquet O. (1983), «L'automobile, un bien banalisé », Économie et statistique, $\mathrm{n}^{\circ}$ 54, pp. 47-55.

Coulmont B. (2011), Sociologie des prénoms, La Découverte, Paris.

Cousteaux A.-S. et Lemel Y. (2004), « Étude de l'Homophilie Socioprofessionnelle à travers l'enquête contacts », Documents de travail du Crest, 2004-10.

Darras (1966), Le Partage des bénéfices : expansions et inégalités en France, Éditions de Minuit, Paris.

Galbraith J.K. (1958), The Affluent Society, Houghton Mifflin, New York.

Gartman D. (1991), « Culture as Class Symbolization or Mass Reification? A Critique of Bourdieu's Distinction ", American Journal of Sociology, 97(2), pp. 421-447.

Gartman D. (2004), «ThreeAges of the Automobile: The Cultural Logics of The Car », Theory Culture

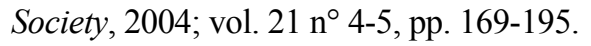

Herpin N. (2001), Sociologie de la consommation, La Découverte, Paris.

Hubert J.-P. et Delisle F. (2010), « L'allongement des déplacements quotidiens contribue à l'émergence d'espaces urbains multipolaires, tandis que la mobilité baisse au centre des grandes agglomérations », Revue du CGDD, décembre, pp. 49-64.

Juillard M. (2007), « Le budget automobile des ménages s'adapte aux prix des carburants », Insee Première, $\mathrm{n}^{\circ} 1159$, octobre.

Le Jeannic T. (1997), « Trente ans de périurbanisation : extension et dilution des villes ». Économie et Statistique ${ }^{\circ} 307$, pp. 21-44.

Kaufmann V., Bergman M.M. et Joye D. (2004), « Motility: Mobility as Capital », International Journal of Urban and Regional Research, vol. 28, $\mathrm{n}^{\circ} 4$, décembre, pp. 745-756.

Le Roux B. et Rouanet H. (2004), Geometric Data Analysis from Correspondence Analysis to Structured Data, Analysis, Kluwer Academic Publisher, Dordrecht-Boston-London.

Lemel Y. (2006), " The Social Positioning of the French According to the EPCV Survey ", Documents de travail du Crest, 2006-14.

Lieberson S. (2000), A matter of taste: How names, fashions, and culture change, Yale University Press.

Lung Y., Chanaron J.-J., Fujimoto T. et Raff D. (éd.) (1999), Coping With Variety: Flexible Productive Systems for Product Variety in the Auto Industry, Ashgate, Aldershot.

Mendras H. (1988), La seconde révolution Française, Gallimard, Paris.

Méot T. (2005), L'industrie automobile en 2004, Insee première, $\mathrm{n}^{\circ} 1036$.

Panzaer J. et Willig R. (1981), « Economies of Scope ", American Economic Review, 71(2), 71(2), pp. 268-272.

Robin M. (2010), « La motorisation des ménages continue de s'accroître au prix d'un vieillissement du parc automobile », Revue du CGDD, décembre, pp. 99-121.

Teece David J. (1980), « Economies of Scope and the Scope of the Enterprise ", Journal of Economic Behavior \& Organization,1(3), 1980, pp. 223-247.

Veblen T. (1970), Théorie de la classe de loisir, Gallimard (édition originale : 1899), Paris. 


\section{DISTRIBUTION DES TROIS PROFILS DE MOBILITÉ SELON QUELQUES VARIABLES} SOCIODÉMOGRAPHIQUES

\begin{tabular}{|c|c|c|c|c|}
\hline & $\begin{array}{c}\text { Profil } 1 \\
\text { Hypo-mobiles }\end{array}$ & $\begin{array}{c}\text { Profil } 2 \\
\text { Hyper-mobiles } \\
\text { à dominante automobile }\end{array}$ & $\begin{array}{c}\text { Profil } 3 \\
\text { Hyper- mobiles } \\
\text { à dominante transports } \\
\text { en commun }\end{array}$ & Ensemble \\
\hline \multicolumn{5}{|l|}{ Sexe } \\
\hline Homme & 47,4 & 45,4 & 53,2 & 46,9 \\
\hline Femme & 52,6 & 54,6 & 46,9 & 53,1 \\
\hline Total & 100,0 & 100,0 & 100,0 & 100,0 \\
\hline \multicolumn{5}{|l|}{ Âge } \\
\hline$<30$ & 9,7 & 11,0 & 15,0 & 11,1 \\
\hline$[30-40[$ & 11,8 & 17,1 & 19,5 & 16,0 \\
\hline$[40-50[$ & 15,4 & 19,8 & 19,0 & 18,5 \\
\hline$[50-60[$ & 21,4 & 23,9 & 23,2 & 23,1 \\
\hline $60<$ & 41,7 & 28,2 & 23,3 & 31,3 \\
\hline Total & 100,0 & 100,0 & 100,0 & 100,0 \\
\hline \multicolumn{5}{|l|}{ Taille de l'unité urbaine de résidence } \\
\hline Commune rurale & 53,8 & 35,6 & 16,3 & 38,3 \\
\hline$<20,000$ habitants & 14,9 & 13,5 & 6,4 & 13,0 \\
\hline [20-100 000 habitants [ & 8,3 & 8,3 & 5,0 & 7,9 \\
\hline 100000 habitants $<$ & 15,6 & 23,8 & 17,9 & 20,9 \\
\hline Paris & 7,5 & 18,8 & 54,4 & 19,9 \\
\hline Total & 100,0 & 100,0 & 100,0 & 100,0 \\
\hline \multicolumn{5}{|l|}{ Catégorie socioprofessionnelle } \\
\hline Agriculteurs & 9,4 & 3,1 & 1,0 & 4,5 \\
\hline Artisans et commerçants & 7,8 & 5,4 & 3,6 & 5,8 \\
\hline Chefs d'entreprises et professions libérales & 0,8 & 2,4 & 3,1 & 2,0 \\
\hline Cadres supérieurs & 6,5 & 18,5 & 29,3 & 16,5 \\
\hline Professions intermédiaires & 17,7 & 25,7 & 25,8 & 23,5 \\
\hline Employés & 29,2 & 26,9 & 19,6 & 26,7 \\
\hline Ouvriers & 25,2 & 14,4 & 11,5 & 17,0 \\
\hline Étudiants & 1,3 & 1,8 & 5,0 & 2,0 \\
\hline Autres inactifs n'ayant jamais travaillé & 2,4 & 1,8 & 1,3 & 1,9 \\
\hline Total & 100,0 & 100,0 & 100,0 & 100,0 \\
\hline \multicolumn{5}{|l|}{ Statut d'activité } \\
\hline Actif & 44,9 & 60,7 & 64,1 & 56,8 \\
\hline Inactif & 55,1 & 39,3 & 35,9 & 43,2 \\
\hline Total & 100 & 100 & 100 & 100 \\
\hline Effectif & 3627 & 8016 & 1573 & 13216 \\
\hline
\end{tabular}

Lecture : 9,7\% des individus du premier profil sont âgés de moins de 30 ans

Champ : ménages possédant au moins un véhicule automobile, France métropolitaine.

Source : enquête nationale Transports et déplacements (ENTD) 2008, Insee. 
